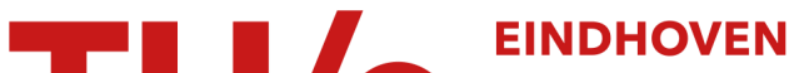 UNIVERSITY OF TECHNOLOGY
}

\section{Streak interactions and breakdown in boundary layer flows}

Citation for published version (APA):

Brandt, L., \& Lange, de, H. C. (2008). Streak interactions and breakdown in boundary layer flows. Physics of Fluids, 20(2), 024107-1/16. [024107]. https://doi.org/10.1063/1.2838594

DOI:

$10.1063 / 1.2838594$

Document status and date:

Published: 01/01/2008

\section{Document Version:}

Publisher's PDF, also known as Version of Record (includes final page, issue and volume numbers)

\section{Please check the document version of this publication:}

- A submitted manuscript is the version of the article upon submission and before peer-review. There can be important differences between the submitted version and the official published version of record. People interested in the research are advised to contact the author for the final version of the publication, or visit the $\mathrm{DOI}$ to the publisher's website.

- The final author version and the galley proof are versions of the publication after peer review.

- The final published version features the final layout of the paper including the volume, issue and page numbers.

Link to publication

\section{General rights}

Copyright and moral rights for the publications made accessible in the public portal are retained by the authors and/or other copyright owners and it is a condition of accessing publications that users recognise and abide by the legal requirements associated with these rights.

- Users may download and print one copy of any publication from the public portal for the purpose of private study or research.

- You may not further distribute the material or use it for any profit-making activity or commercial gain

- You may freely distribute the URL identifying the publication in the public portal.

If the publication is distributed under the terms of Article 25fa of the Dutch Copyright Act, indicated by the "Taverne" license above, please follow below link for the End User Agreement:

www.tue.nl/taverne

Take down policy

If you believe that this document breaches copyright please contact us at:

openaccess@tue.nl

providing details and we will investigate your claim. 


\title{
Streak interactions and breakdown in boundary layer flows
}

\author{
Luca Brandt ${ }^{1, a)}$ and H. C. de Lange ${ }^{2}$ \\ ${ }^{1}$ Linné Flow Centre, KTH Mechanics, SE-100 44 Stockholm, Sweden \\ ${ }^{2}$ TUE Mechanical Engineering, 5600 MB Eindhoven, The Netherlands
}

(Received 5 October 2007; accepted 8 December 2007; published online 28 February 2008)

\begin{abstract}
The objective of this paper is to show that the interaction of streamwise velocity streaks of finite length can lead to turbulent breakdown in the flat-plate boundary layer flow. The work is motivated by previous numerical and experimental studies of transitional flows where the high-frequency oscillations leading to turbulence are seen to form in the region of strongest shear induced by streaks in relative motion. Therefore, a model for the interaction of steady and unsteady (i.e., slowly moving in the spanwise direction) spanwise periodic streaks is proposed. The interaction of two subsequent streaks is investigated for varying collision parameters. In particular, the relative spanwise position and angle are considered. The results show that the interaction is able to produce both a symmetric and asymmetric breakdown without the need for additional random noise from the main stream. Velocity structures characteristic of both scenarios are analyzed. Hairpin and $\Lambda$ vortices are found in the case of symmetric collision between a low-speed region and an incoming high-speed streak, when a region of strong wall-normal shear is induced. Alternatively, when the incoming high-momentum fluid is misaligned with the low-speed streak in front, single quasi-streamwise vortices are identified. Despite the different symmetry at the breakdown, the detrimental interaction involves for both cases the tail of a low-speed region and the head of a high-speed streak. Further, the breakdown appears in both scenarios as an instability of three-dimensional shear layers formed between the two streaks. The streak interaction scenario is suggested to be of relevance for turbulence production in wall-bounded flows. (C) 2008 American Institute of Physics.
\end{abstract}

[DOI: $10.1063 / 1.2838594]$

\section{INTRODUCTION}

The occurrence and breakdown of streamwise velocity streaks have been identified as key elements in the transition to turbulence and in the dynamics of turbulence in wallbounded shear flows. Indeed, low-speed streaks are observed at the late stages of different transition scenarios, and streak breakdown is one of the phases of the regeneration cycle which is believed to sustain a turbulent flow. The streaks are perturbations elongated in the streamwise direction consisting of negative/positive streamwise velocity perturbations, which induce localized regions of decelerated/accelerated flow. Their generation is due to the presence of streamwise vorticity or spanwise-dependent vertical motions in shear flows (the so-called lift-up effect ${ }^{1}$ ), and the following large amplification of the streaks can be explained successfully by the theory of nonmodal growth. ${ }^{2}$ In the presence of high levels of background disturbances, e.g., turbulent wallbounded flows and bypass transition, many streaks are forming randomly in time and space, and interactions among them are likely to occur. The aim of the present work is to investigate such interactions and show when and how they can lead to turbulent production or transition to turbulence.

The low- and high-speed streaks are always surrounded by regions of large shear both in the wall-normal and in the spanwise directions. The corresponding inflectional velocity distributions are thus able to support strong instabilities of an

\footnotetext{
${ }^{a)}$ Electronic mail: luca@ mech.kth.se.
}

inviscid nature. This has led many researchers to consider the instability of streamwise streaks in wall-bounded shear flows in simple configurations, the most common being the assumptions of steady, symmetric, and spanwise periodic streaky flows. The experiments by Swearingen and Blackwelder ${ }^{3}$ were the first to document the emergence of streaks with inflectional profiles due to the formation of Görtler vortices in the boundary layer over a concave wall. This investigation demonstrated that time-dependent fluctuations appear in the flow either in a spanwise symmetric (varicose) or antisymmetric (sinuous) pattern with respect to the underlying streak. The varicose perturbations have been correlated with the wall-normal inflection points, while the sinuous oscillations are related with the spanwise inflectional profile. The latter were found to be the fastest growing.

The instability of steady and periodic streaks of infinite length arising from the transient growth of streamwise vortices was first studied in channel flows. ${ }^{4-7}$ These studies confirmed that the instability is of inflectional type and that the dominating instability appears as spanwise (sinuous) oscillations of the streaks. For zero-pressure-gradient boundary layers, the spatial response of a single low-speed streak submitted to a time-harmonic excitation of sinuous or varicose type has been more recently examined experimentally by Asai $e t$ $a l .{ }^{8}$ The growth of the sinuous mode was observed to evolve into a train of quasi-streamwise vortices with vorticity of alternate sign. By contrast, the varicose mode led to the formation of hairpin structures made up of a pair of counterrotating vortices. Numerically, the instability and breakdown 
of streaks for flat-plate boundary layers was investigated in Refs. 9-11.

From these numerical simulations, it was possible to investigate the late stages of the breakdown triggered by the streak instability and identify the relevant flow structures. In the case of sinuous breakdown, the formation of quasistreamwise vortices located on the flanks of the low-speed streak in a staggered pattern is observed. For symmetric perturbations, hairpin or $\Lambda$ vortices are found. The breakdown mechanisms and the corresponding flow structures are very similar to those observed for turbulent flows. Studies on the instability of a base flow generated by the superposition of a turbulent mean flow and steady streaky structures can be found in Refs. 5 and 12. Moreover, the flow structures observed in the numerical simulations in limiting conditions for the turbulence to be self-sustained ${ }^{13}$ are also similar to those observed when the sinuous streak instability is studied. Hairpin vortices, associated with a symmetric distribution of the perturbations growing on the streak, are also found in turbulent flows. ${ }^{14,15}$

The present work considers the interaction of streaks of finite length and varying relative position. It differs from prior studies of streak instability mechanisms, where the breakdown and associated vortices are generated via growth of small-amplitude disturbances on a steady base flow: Here, the initial condition is unsteady with finite-amplitude velocity disturbances and does not contain an identifiable base flow. The work is motivated by observations of transition to turbulence in flat-plate boundary layers subject to high levels of free-stream turbulence both from the direct numerical simulations ${ }^{16}$ and the experiments ${ }^{17-19}$ performed in our groups in Stockholm and Eindhoven. This so-called bypass transition scenario is characterized by the appearance inside the boundary layer of large amplitude streaks. As the streaks grow downstream, they undergo wavy motions that precede the breakdown into regions of intense randomized flow, namely turbulent spots. The numerical simulations of transition in Ref. 16 enabled the identification of the structures at the streak breakdown. These vortical structures are very similar to those predicted both for sinuous and varicose modes in the simplified setting of the secondary instability of steady periodic streaks. The same breakdown scenarios are identified in the flow visualization from the water channel experiments in Ref. 17. These studies also showed the importance of unsteadiness and interaction among streaks. These are found to be an important triggering mechanism of the streak breakdown: High-frequency oscillations are seen to form and grow in those regions of strongest shear that are induced by streaks in relative motion. Streaks appear randomly and elongate in the streamwise direction while growing; it is observed that their leading and trailing edge travel at different velocities (and also that some streaks can move faster than others). These facts lead to streak interactions, most commonly the head of later generated streaks colliding with the tail of earlier generated streaks. A necessary condition for this to happen is a sufficiently high streak-creation rate. Therefore, these collisions are mainly observed for moderate-to-high levels of free-stream turbulence; indeed, in the simulation by Ovchinnikov et al. ${ }^{20,21}$ with higher levels of free-stream turbulence, rapid perturbation growth at the tails of shorter regions of high- and low-momentum fluid is found as the main characteristic of spot precursors. The relevance of such streak interactions was first suggested by Johansson et $_{\text {al. }}{ }^{22}$ and Landahl ${ }^{23}$ for the case of near-wall turbulent streaks. In the former study, the development of the strong three-dimensional shear layers associated with approaching streaks was analyzed by conditional sampling. Note that also Wu and Choudhari ${ }^{24}$ showed that the unsteadiness of the Klebanoff modes (or streaks) plays a crucial role. These authors found instability modes that would not have been present if streaks of the same amplitude were treated as steady.

An interesting issue related to streak breakdown in transition in boundary layers subject to high levels of free-stream turbulence is the existence of a critical threshold amplitude. Further analysis of the experiments in Refs. 17 and 18, presented at the European Fluid Mechanics Conference 6 (EFMC6) in June of 2006, reveals that the streak amplitude is not the relevant parameter for the breakdown: Streaks of the same amplitude do not always undergo breakdown. Linear stability analysis predicted threshold streak amplitudes of $35 \%$ in the plane channel ${ }^{6}$ and $26 \%$ in boundary-layer flow, ${ }^{9}$ values considered higher than those one would expect from experiments. However, Waleffe ${ }^{25}$ showed the subcritical nature of streak instability in channel flows. The results by Hoepffner $e t a l .{ }^{26}$ for (infinite, periodic) streaks in boundary layers demonstrated the possibility of strong transient disturbance growth on the streak, which may be responsible for subcritical breakdown. In the proposed collision scenario, the amplitude of the streak becomes indeed a parameter of secondary importance.

In this paper, we present for the first time a model for the interaction between streaks, assumed spanwise periodic but of finite length and small frequency, i.e., slowly moving in the spanwise direction. It will be shown that these collisions are able to trigger the streak breakdown without any additional random noise. The corresponding structures at the breakdown will be analyzed. In the case of turbulent boundary layers and flows exposed to free-stream turbulence, the presence of external noise would certainly affect the breakdown, most likely anticipating it. However, the influence of ambient disturbances is not considered here. The aim of the paper is to propose and investigate a mechanism for turbulence production, the streak collision, which is believed to be important when streaks are induced randomly in time and space.

\section{NUMERICAL METHOD AND STREAK INTERACTION MODEL}

\section{A. Numerical scheme}

The simulation code employed for the present computations was developed at KTH Mechanics ${ }^{27}$ and uses spectral methods to solve the three-dimensional, time-dependent, incompressible Navier-Stokes equations. The code has now been used for more than a decade for the direct numerical simulation of both transitional and turbulent flows. Detailed 
descriptions of the algorithm adopted for spatially evolving boundary layers can also be found in Ref. 10.

To correctly account for the downstream boundary-layer growth, a spatial technique is necessary. This requirement is combined with the periodic boundary condition in the streamwise direction by the implementation of a "fringe region," similar to that described by Bertolotti et al. ${ }^{28}$ In this region, the flow is smoothly forced to the prescribed inflow velocity field. This is normally a boundary-layer profile with the addition of the inflow disturbance one wishes to examine. For further details on this technique, see Refs. 27 and 29. To set the free-stream boundary condition at $y=y_{\max }$, a generalization of the boundary condition used by Malik et al. ${ }^{30}$ is implemented.

\section{B. Streak generation}

In the simulation presented here, the zero-pressuregradient boundary layer flow is considered. The inflow is at Reynolds number $\operatorname{Re}_{\delta_{0}^{*}}=U_{\infty} \delta_{0}^{*} / \nu=344.16$, where $\nu$ is the fluid viscosity, and the quantities $U_{\infty}$ and $\delta_{0}^{*}$, the free-stream velocity and displacement thickness at the inlet, will be used to nondimensionalize the dependent variables throughout the paper. The independent variables $x, y$, and $z$ will denote the streamwise, wall-normal, and spanwise coordinate, respectively, with dimensionless time $t=\bar{t} U_{\infty} / \delta_{0}^{*}$.

The complete velocity vector field obtained with the linear code developed by Levin and Henningson ${ }^{31}$ is used to force the desired streaky perturbation at the inflow of the computational domain. These streaks are introduced in the fringe region by adding them to the inflow Blasius profile. The streaks considered are optimally growing perturbations, solution of the linearized boundary-layer equations, and are characterized by the spanwise wavenumber $\beta$, the time frequency $\omega$ (low in order to obey the boundary-layer approximation), and the streamwise location of maximum amplitude. The value $\beta=0.542$ will be considered here. This value, corresponding to a streak periodicity of 11.54 , has been chosen in order to approximately reproduce the spacing observed at the breakdown stages in the experiments. ${ }^{17}$ Five equidistant circular frequencies in the range $\omega \in\left[0,1.4 \times 10^{-2}\right]$ are examined; the selected range corresponds to values of about $0-12 \mathrm{~Hz}$ if one wishes to compare with the experiments in Ref. 32. In the latter work, energy spectra for the streaky flow, showing highest energy in the range $0-8 \mathrm{~Hz}$, are reported. Note that low-frequency streaks appear as oblique waves, i.e., as tilted structures slowly moving in the spanwise direction.

For the studies on the interaction mechanisms, the streak velocity profiles are chosen to give a maximum amplitude of about $A_{S}=0.28 U_{\infty}$ at $x=200$, where $A_{S}$ is defined as half the peak-to-peak difference between low- and high-speed regions. By contrast, in the analysis of the amplitude effect, a streak amplifying over a longer distance $(x=350)$ is selected in order to consider the possibility of collisions at later times (see below). An example of the inflow streak profiles is reported in Fig. 1, where the background color indicates streamwise vorticity and the lines the streamwise velocity perturbation: The inflow perturbation consists mainly of

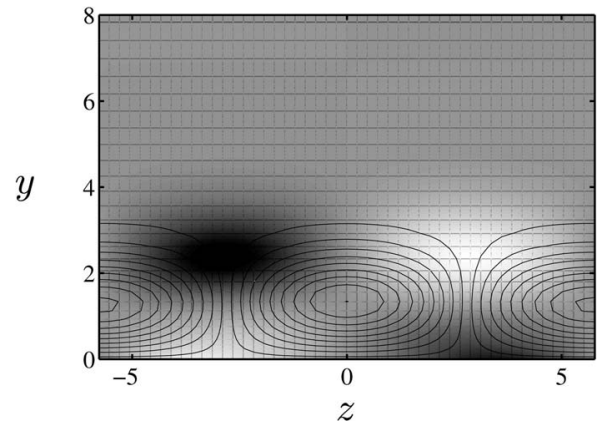

FIG. 1. The inflow streaky perturbation, $x=0$, for $\beta=0.542$ and $\omega=0$. The background depicts the streamwise vorticity with shading ranging from black to white for negative and positive values, respectively. The maximum value of the vorticity is $\omega_{x}=0.0124$. The lines display streamwise velocity perturbation. Minimum contour line -0.2125 , contour spacing 0.025 . Dashed lines are used for negative values.

streamwise velocity, with weak decaying streamwise vorticity. The streamwise development of the streak amplitude is displayed in Fig. 2 for the five values of $\omega$, which will be considered in Sec. III.

\section{Interaction parameters}

In the present study, the collision between the trailing edge of a downstream (earlier generated) streak and the leading edge of an upstream (later) streak is considered. The flow is initialized by prescribing the spatially developing (zeropressure gradient) Blasius profile $u_{\text {blas }}(x, y)$. Added to this, two streaky disturbances of finite length are generated at the inflow of the computational domain by forcing them in the fringe region only during a prescribed time interval. The control parameters governing the following evolution are therefore (i) the time interval between the tail of the first streak and the head of the second one, in other words the initial distance between the two colliding structures; (ii) the relative spanwise phase, i.e., the relative position between the downstream low-speed region and the upstream high-speed region; and (iii) the angle between the axis of the two colliding streaks, being $0^{\circ}$ for two streamwise aligned streaks of zero frequency (see Fig. 3).

The function used to modulate the amplitude of the two

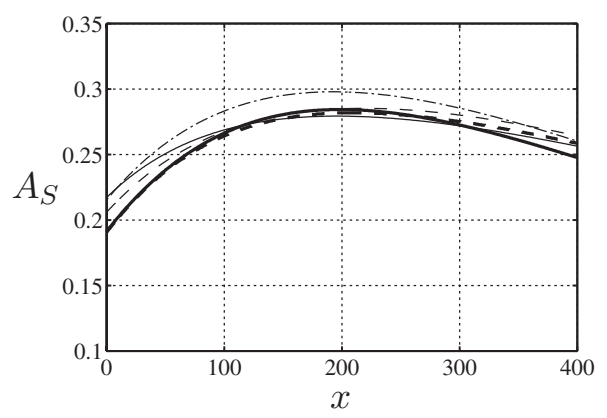

FIG. 2. Streamwise growth of the streak amplitude $A_{S}$ computed from the linear boundary-layer equations. The inflow amplitude is set to the value used in the simulations of breakdown. (-): $\omega=0 ;(---): \omega=3.44 \times 10^{-3}$ $(---): \omega=6.88 \times 10^{-3} ;(-): \omega=1.032 \times 10^{-2} ;(---): \omega=1.37 \times 10^{-2}$. 


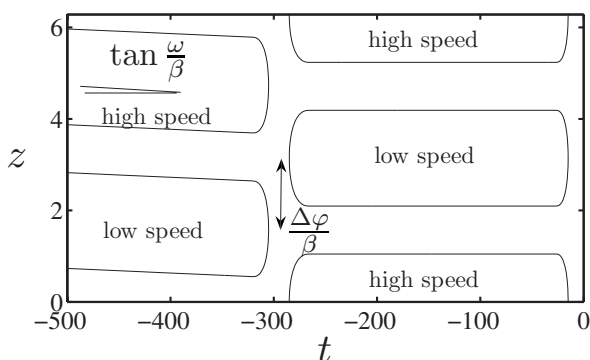

FIG. 3. Sketch of the parameters defining the streak interactions: The relative spanwise phase $\Delta \varphi$ and the angle between the axis of the two colliding streaks varied through the frequency $\omega$ of the upstream streak.

colliding streaks, which was actually implemented to provide the desired finite length of the inflow perturbation, is

$$
f(t)=A_{S}\left[S\left(\frac{t-t_{\mathrm{st}}}{t_{\text {rise }}}\right)-S\left(\frac{t-t_{\mathrm{end}}}{t_{\text {fall }}}\right)\right]
$$

where $A_{S}$ is the streak amplitude and $S(a)$ is a smooth function rising from zero for negative $a$ to 1 for $a \geqslant 1$,

$$
S(a)= \begin{cases}0 & a \leqslant 0 \\
1 /\left[1+\exp \left(\frac{1}{a-1}+\frac{1}{a}\right)\right] & \begin{array}{l}
0<a<1 . \\
1
\end{array} \\
a \geqslant 1\end{cases}
$$

In expression (1), $t_{\mathrm{st}}$ and $t_{\mathrm{end}}$ are the initial and final time for the generation of the streak, with $t_{\text {rise }}$ and $t_{\text {fall }}$ the "rise" and "fall" interval of the damping function. The following values are chosen for the simulations presented here, if not otherwise stated, where the superscripts 1 and 2 refer to the downstream and upstream streak, respectively. $t_{\mathrm{st}}^{1}=0, t_{\text {end }}^{1}$ $=260, t_{\text {rise }}^{1}=50$, and $t_{\text {fall }}^{1}=30 ; t_{\mathrm{st}}^{2}=285, t_{\text {end }}^{2}=800, t_{\text {rise }}^{2}=30$, and $t_{\text {fall }}^{2}=40$.

The time interval of generation of the first streak $\left(t_{\text {end }}^{1}\right.$ $\left.-t_{\mathrm{st}}^{1}\right)$ is merely chosen large enough to develop the streak profile further downstream. In our simulations, the gap width $\left(t_{\mathrm{st}}^{2}-t_{\mathrm{end}}^{1}\right)$ has been shown to have little influence on the results as they will be presented. Note that elongation of the streaks is observed mainly in the growth phase and, therefore, a longer initial distance would only require a larger amplification distance but lead to the same interaction only further downstream (as verified in our simulations). For a smaller gap width, the breakdown develops somewhat faster and more violently. Therefore, the time interval between the generation of the two streaks has been kept constant for most of the results presented here. A parameter study of the interaction between streaks of different relative spanwise phase and different frequency (or obliqueness) is presented.

The streak profiles extracted from the linearized boundary layer code ${ }^{31}$ are normalized so as to have the low-speed region at the center of the computational domain $(z=0)$ (cf. Fig. 3). The relative spanwise position is therefore adjusted by adding for the upstream perturbation a phase shift $\varphi$ to the complex exponential factor of the inflow complex velocity field

$$
\exp [i(\beta z-\omega \widetilde{t})]
$$

where $\tilde{t}=t-t_{0}$ and $t_{0}=\left(t_{\text {end }}^{1}+t_{\text {st }}^{2}+t_{\text {rise }}^{2}\right) / 2$. The angular frequency $\omega$ defines the angle between the streak and the streamwise direction, therefore the angle between the two interacting streaks is proportional to the difference in frequency, $\omega^{1}-\omega^{2}$.

\section{Numerical resolution and computational domain}

The numerical simulations are performed using two different computational domains and corresponding resolution. All the cases are first simulated in a box that measures $500 \times 20 \times 11.54$ in units of $\delta_{0}^{*}$ in the streamwise, wall-normal, and spanwise directions, respectively, where $512 \times 73 \times 48$ grid points are used. A check on the accuracy of the simulations is obtained by comparing with the results from computations with higher resolution. The latter use a shorter box, $400 \delta_{0}^{*}$, and a larger number of modes, $640 \times 81 \times 64$. For both domains, the length of the fringe region, used to enforce the inflow streaky profile, is $50 \delta_{0}^{*}$.

\section{SCENARIOS WHEN VARYING THE SPANWISE PHASE}

The effect of varying the relative position of the two interacting perturbations is considered first: The spanwise phase of the streak profile with $\omega=0$ is changed from 0 to $\pi$. Note that for a shift of $\pi$, the head of the second high-speed streak interacts with the tail of the first low-speed streak, while for a zero phase shift, the streak profile is merely interrupted during $\left(t_{\mathrm{st}}^{2}-t_{\text {end }}^{1}\right)$. When varying the relative spanwise phase, three different scenarios are observed. For low values of $\Delta \varphi$, the two subsequent streak structures join. The initial gap between both streaks is eventually bridged. For $\Delta \varphi \approx \pi$, the upstream high-speed streak collides almost symmetrically with the downstream low-speed streak. At intermediate values of $\Delta \varphi \approx \pi / 2$, the interaction also leads to breakdown. In this case, the flow fields reveal the asymmetry of the collision. As showcases, the spanwise shifts of $\pi$ and $\pi / 2$ are examined. A three-dimensional view of the initial configuration of the interacting structures in terms of streamwise velocity and vorticity can be deduced from Figs. 10(a) and $17(\mathrm{a})$.

\section{A. Breakdown initiation}

To determine at which time the difference in the interaction mechanism for $\Delta \varphi$ equals $\pi / 2$ and $\pi$ starts, the development of the averaged spanwise velocity $\langle w\rangle$ is considered,

$$
\langle w\rangle \equiv \frac{1}{L_{x} L_{z}} \int_{L_{x}} \int_{L_{z}} w(x, 2, z) d z d x .
$$

The evolution of this quantity is chosen to represent the amplification of the high-frequency modes leading to breakdown since it is initially very weak. In Fig. 4(a), $\langle w\rangle(t)$ is presented for both phase shifts, and the same streak amplitude, when both lead to breakdown. At $t=t_{\mathrm{st}}^{2}$, the arrival of 
a)

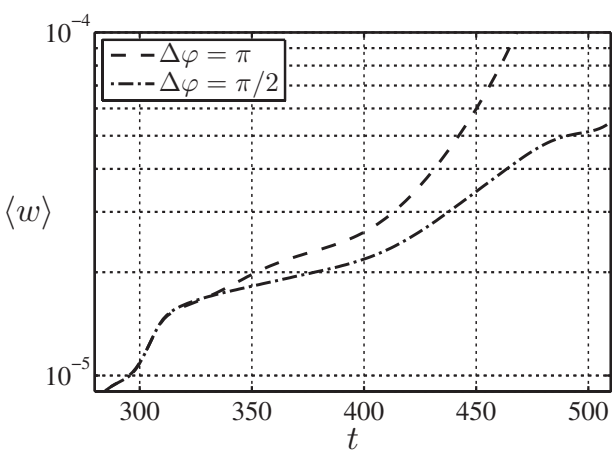

b)

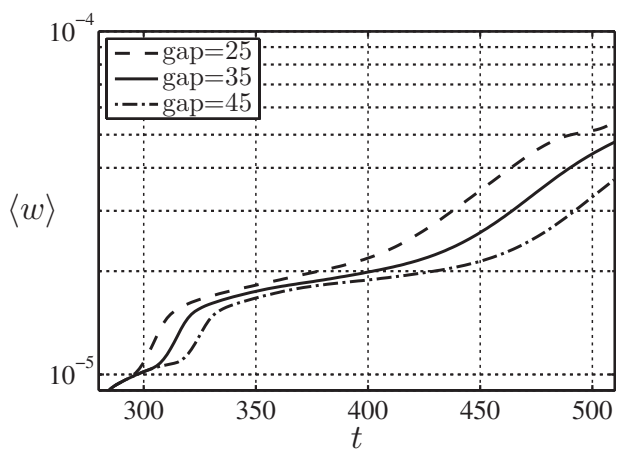

FIG. 4. (a) Time behavior of the averaged spanwise velocity $\langle w\rangle$ for the symmetric and asymmetric breakdown of two straight streaks $(\omega=0)$ obtained with relative spanwise phase of $\Delta \varphi=\pi / 2$ and $\pi$, respectively. (b) Evolution of $\langle w\rangle$ for different initial distances between the colliding streaks, $t_{\mathrm{st}}^{2}-t_{\mathrm{end}}^{1}=25,35$, and 45 at $\Delta \varphi=\pi / 2$.

the second streak initially leaves $\langle w\rangle$ unaffected. However, after a short time, the tail of the first streak and the head of the one upstream start to interact. This manifests as a slight increase in $\langle w\rangle$ at $t \approx 320$. For $\Delta \varphi=\pi / 2$, the averaged perturbation $\langle w\rangle$ is at first slightly higher, however its increase is slower for $t>350$. It appears that the time to transition measured from the initial interaction to breakdown is longer for the asymmetric case. The results show that at the initial stages of the interaction (for $t \approx 350$ ), the difference between different scenarios is already recognizable.

In Fig. 4(b), the evolution of $\langle w\rangle$ is presented for increasing gap widths $\left(t_{\mathrm{st}}^{2}-t_{\text {end }}^{1}\right)$ and a phase shift $\Delta \varphi=\pi / 2$. The results show that increasing the gap width leads to a delay in the collision time, the traces of breakdown for the three gap widths being very similar. Besides the delayed beginning of the interactions, one can notice that the growth of $\langle w\rangle$ at the later stages of breakdown is slightly slower for the simulation with a time delay of 45 . This can be explained by the fact that when breakdown occurs further downstream, the streaks have had the time to start their decay and their instability is weaker.

\section{B. Symmetric breakdown}

In Fig. 5, the breakdown process for a phase shift of $\Delta \varphi=\pi$ is presented by surfaces of constant streamwise velocity perturbation, where high-speed fluid is indicated by a light (gold) color and low-speed fluid by a dark (blue) color. It can be seen that the upstream streaky pattern collides head-on with the low-momentum fluid that remains after the
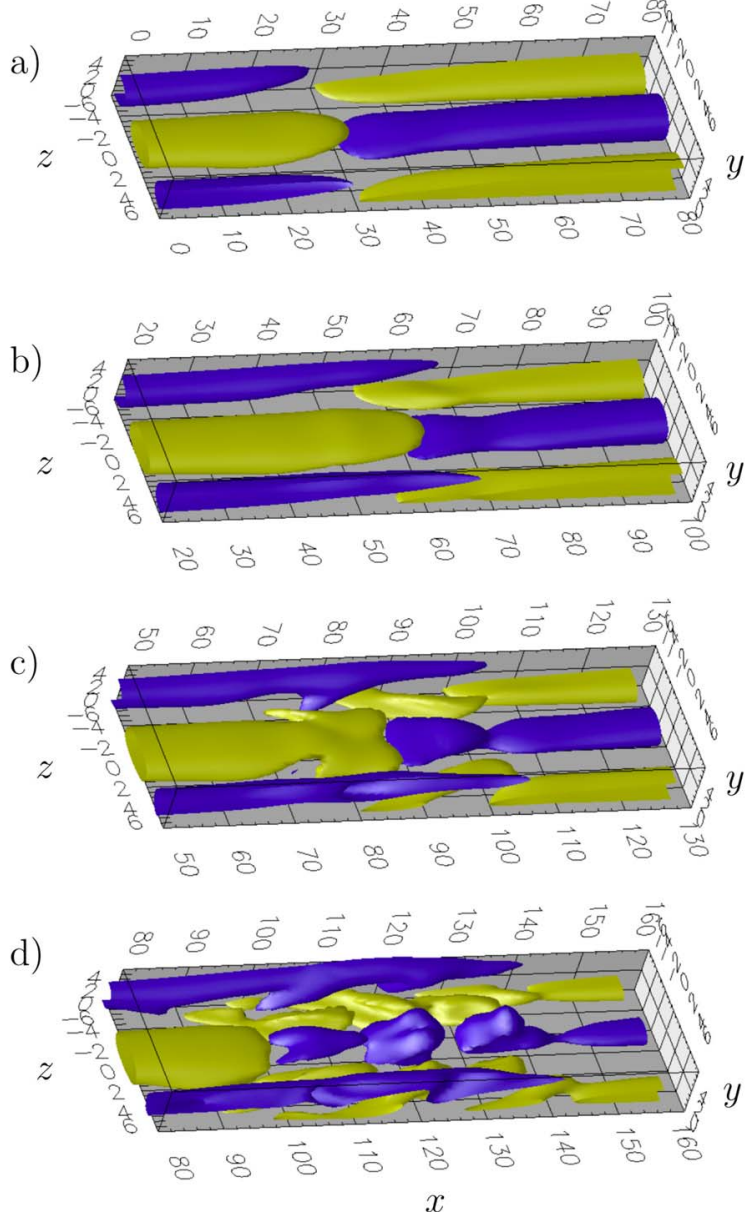

FIG. 5. (Color online) Evolution of the streaky structures during the symmetric breakdown, $\Delta \varphi=\pi$, depicted at time $t=350,400,450$, and 500 [(a)(d)]. The light (gold) color displays surfaces of positive streamwise velocity, whereas low-speed fluid is depicted in a dark (blue) color. The values shown are $u-u_{\text {blas }}= \pm 0.1$ in (a) and (b), $u-u_{\text {blas }}= \pm 0.12$ in (c), and $u-u_{\text {blas }}$ $= \pm 0.15$ in $(\mathrm{d})$.

downstream low-speed streak. At $t=400$, a spanwise symmetric deformation of the two interacting areas is observed. This is followed by the formation of islands of low-speed fluid, positioned symmetrically around the tail of the downstream perturbation and originating by the destruction of the low-speed streak. The number of these structures is increasing during the breakdown [see Fig. 5(d)]. Similar splitting of the low-speed streak is also found in the experimental results in Refs. 8 and 17 and the numerical results in Ref. 16.

In Fig. 6, velocity contours of the streamwise velocity perturbation $u(x, y, 0)-u_{\text {blas }}(x, y)$ are presented in the wallnormal plane in the middle of the streak $(z=0)$ at $t=350$, 400 , and 450. At the interaction, the low-speed fluid close to the surface acts as an obstacle to the incoming high-speed fluid. This leads to the formation of a three-dimensional surface of strong shear, associated with inflectional wall-normal profiles, tilted downstream away from the wall. This area is elongating in the streamwise direction as the interaction proceeds since fluid in the upper part of the boundary layer is convected at higher speeds. At $t=400$ a waviness of the shear region, which is a sign of a developing instability, has become apparent. This wave has a length of about 10 in the 
$y$

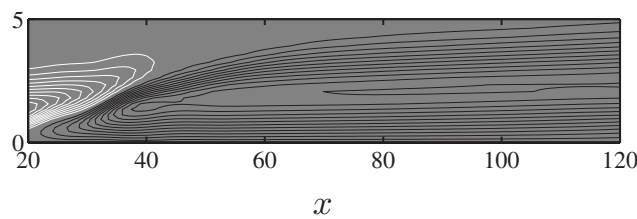

$y$
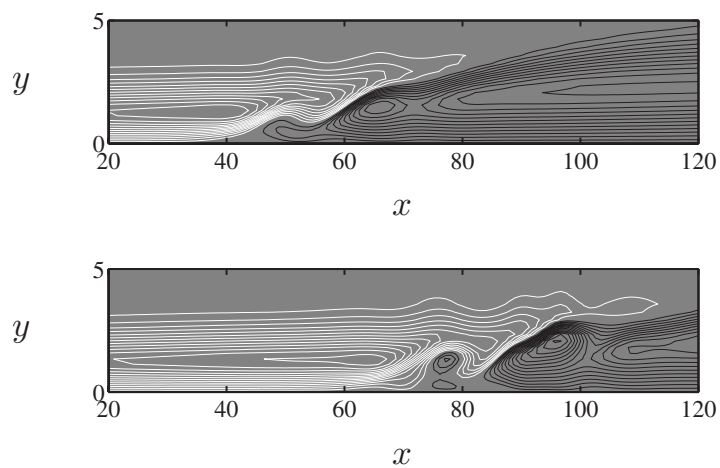

FIG. 6. The shear-layer instability developing during the symmetric streak interaction $(\Delta \varphi=\pi)$, depicted by negative (black) and positive (white) contour levels, is the streamwise perturbation velocity $u-u_{\text {blas }}$ in the $z=0$ plane at time $t=350,400$, and 450 . Contour spacing is 0.02 .

streamwise direction and extends from the wall-surface to about $y=1.5$. As time evolves, the structure breaks down into patches of low- and high-speed fluid as shown in the plot pertaining to $t=450$. In the plane $z=\pi / \beta$, where the downstream high-speed streak is approached by the upstream lowspeed streak, the stretching of the interaction area observed half a wavelength apart is also found (not shown here). In this case, however, the interacting shear region remains far more stable. Only at $t=450$ do the first signs of small-scale motions, a result of the spanwise propagation of the instability at the center plane $z=0$, become visible.

The unstable small-scale motions, originating from the streak interaction and responsible for the streak disruption observed in Fig. 5, can be visualized by looking at the wallnormal and spanwise velocity developing in the form of wave packets. Top views of surfaces of constant values of the latter perturbation velocities, $v-v_{\text {blas }}$ and $w$, are displayed at $t=450$ in Fig. 7, where the low- and high-speed streaks are also depicted for reference. It can be seen that while the wall-normal velocity appears in a pattern symmetric with respect to the underlying streak, its spanwise counterpart is antisymmetric in the spanwise direction, as one would expect from symmetric varicose streak instability. As the instability is triggered, a wave packet of positive and negative wallnormal motions is observed in the region where the two streaks interact. It can also be noted that the islands of lowspeed fluids, more clearly visible in Fig. 5, are associated with negative wall-normal velocity in the front and negative velocity at the rear. The spanwise velocity appears also as regular patches of negative and positive velocity positioned around $z=0$, where the high-speed region upstream is reaching the tail of the low-speed streak in front. The largest contributions are relative to velocity pointing toward the centerline; regions of alternating sign are also present, however the outwards spanwise motions are weaker. This is in contrast to a)

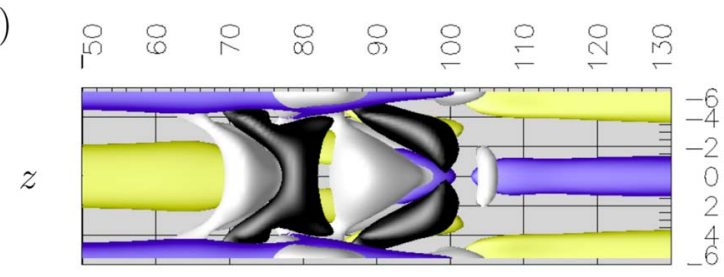

b)

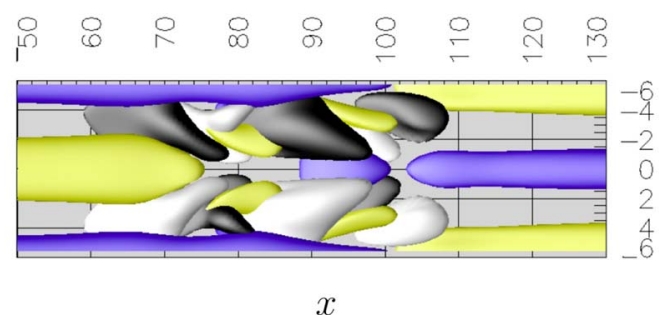

FIG. 7. (Color online) The high-frequency perturbation growing during the symmetric streak interaction, displayed at $t=450$. In both figures, the light (gold) color displays surfaces of positive streamwise velocity $\left(u-u_{\text {blas }}\right.$ $=0.15)$, whereas low-speed fluid $\left(u-u_{\text {blas }}=-0.15\right)$ is depicted in a dark (blue) color. (a) Surface of constant wall-normal velocity perturbation $v$ $-v_{\text {blas }}= \pm 0.01$. (b) Surface of constant spanwise velocity perturbation $w$ $= \pm 0.02$, with black for negative values and white for positive values.

what is observed in the case of the varicose instability of steady infinite streaks, where the positive and negative spanwise velocities have the same intensity. ${ }^{11}$ At $t=450$, the main patches stretch over a length of about 40 , while they reach a distance from the wall of $y \approx 3$.

A three-dimensional view of the vortical structures characteristic of this transition scenario is reported in Fig. 8, both with and without the underlying streaks to indicate their relative position. Vortical structures are identified by isosurfaces of negative $\lambda_{2}$, the second eigenvalue of the Hessian of the pressure. ${ }^{33}$ As observed in the case of the varicose instability of one steady streak ${ }^{8,11}$ and from the simulations of a transition induced by free-stream turbulence, ${ }^{16}$ the typical vortical structures consists of $\Lambda$ structures pointing downstream and upstream alternatively, the latter referred to as $V$ vortices in Ref. 16. Both the $\Lambda$ and $V$ structures are tilted in the wall-

a)

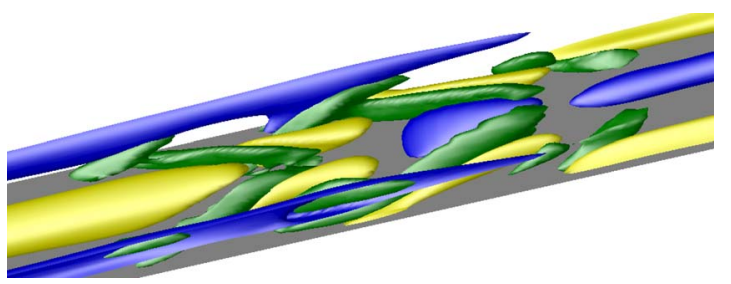

b)

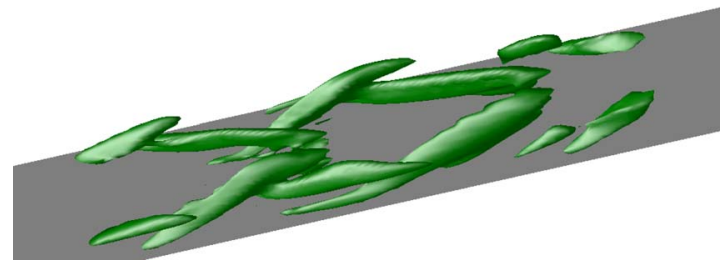

FIG. 8. (Color online) Vortical structures characteristic of the symmetric breakdown at $t=450$. In (a) dark (blue) surfaces represent low-streamwise velocity $\left(u-u_{\text {blas }}=-0.15\right)$ while light gray (gold) surfaces depict high-speed regions $\left(u-u_{\text {blas }}=0.15\right)$. Gray (green) represents negative values of $\lambda_{2}$, used to identify vortical structures. 


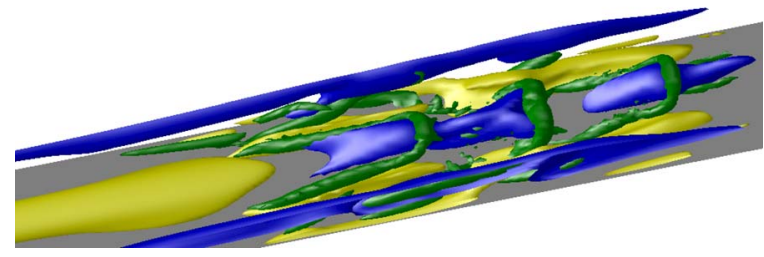

FIG. 9. (Color online) Vortical structures at the late stages of the symmetric breakdown, $t=520$. Dark (blue) surfaces represent low-streamwise velocity $\left(u-u_{\text {blas }}=-0.17\right)$ while light gray (gold) surfaces depict high-speed regions $\left(u-u_{\text {blas }}=0.17\right)$. Gray (green) represents negative values of $\lambda_{2}$.

normal direction: The tip of the $\Lambda$ vortices is located farther away from the wall than the quasi-streamwise legs, whereas in the case of the $V$ vortices the tip is the part located closest to the wall. The streamwise vortices (legs) are formed well before the hairpin heads. In this highly three-dimensional flow, the spanwise head is more likely produced by local twisting of vortex lines and roll-up induced by the streamwise legs. This is in good agreement with previous studies on the breakdown of steady and unsteady streaks. ${ }^{11,16}$ Note also more clearly in the close-up in Fig. 8 the splitting of the interacting streaks, cf. Ref. 8. As the instability grows downstream, only the $\Lambda$ vortices are amplifying leading to the formation of a train of hairpin vortices, as shown by the vortical structures at $t=520$ in Fig. 9. Conversely, the $V$ structures disappear at the breakdown. This shows the amplification of only the streamwise vorticity, which can sustain the streak and induce stronger mean shear. At these later stages, the tip of the hairpin vortices becomes aligned to the wall-normal direction, forming a ring-like structure similar to those observed in several previous studies on laminarturbulent transition (see, e.g., Ref. 34).

To better understand the generation of streamwise vorticity $\omega_{x}$, the evolution of this quantity during the interaction is displayed in Fig. 10. Note that the streamwise vorticity is initially very weak, the interacting structures mainly consisting of streamwise velocity perturbation. $\omega_{x}$ is amplifying in the form of a wave packet with regions of positive and negative vorticity alternating in an antisymmetric pattern. The production of vorticity is due to the tilting of the wall-normal and spanwise vorticity associated with the streamwise velocity variations created by the streaks. Responsible of the tilting are the high-frequency (short streamwise wavelength) wall-normal and spanwise velocity patches developing at the interaction. This is clearly shown in Fig. 11, where the tilting term appearing in the streamwise vorticity equation,

$$
\frac{\partial u}{\partial z} \frac{\partial v}{\partial x}-\frac{\partial u}{\partial y} \frac{\partial w}{\partial x}
$$

and the streamwise vorticity are displayed at time $t=400$. Evidence for the tilting can also be deduced by comparing the patterns in Figs. 5 and 10. (see also Refs. 10 and 35). When considering the two separate contributions in the tilting term reported above, it is found that production from tilting of the spanwise vorticity $\partial u / \partial y$ is more than one order of magnitude larger than that of its wall-normal counterpart. At the later stages of the breakdown, $t>450$, the correlation

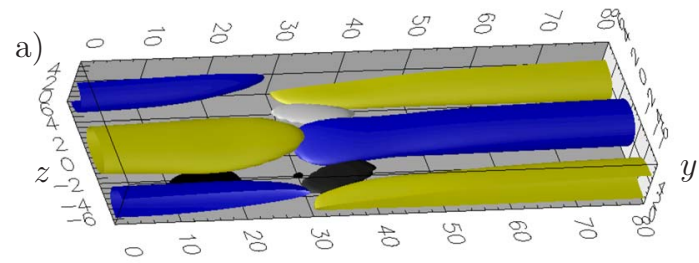

b)
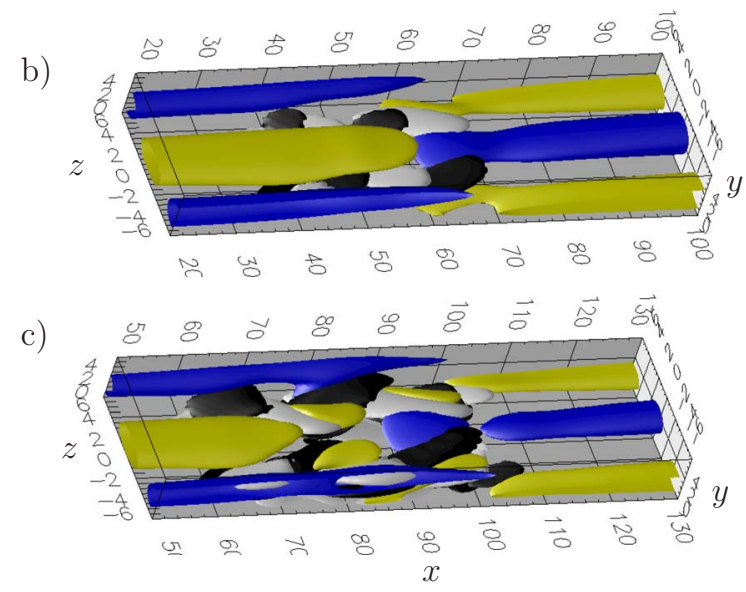

FIG. 10. (Color online) Evolution of the streamwise vorticity during the symmetric breakdown, $\Delta \varphi=\pi$, depicted at time $t=350$, 400, and 450 [(a)(c)]. Positive and negative vorticity are represented in white and black, respectively. The light (gold) color displays surfaces of positive streamwise velocity, whereas low-speed fluid is depicted in a dark (blue) color. The values shown are $\omega_{x}= \pm 0.06 ; u-u_{\text {blas }}= \pm 0.1$ in (a), $u-u_{\text {blas }}= \pm 0.12$ in (b), and $u-u_{\text {blas }}= \pm 0.15$ in $(\mathrm{c})$.

between streamwise vorticity and the vortex-stretching term $\partial u / \partial x$ becomes significant. This is shown by the crossstream cut in Fig. 12, where the contour levels indicate $\partial u / \partial x$ and the background color streamwise vorticity at $x=96$ and $t=450$. As noted by Schoppa and Hussain, ${ }^{36}$ vortex stretching is responsible for the collapse of streamwise

a)

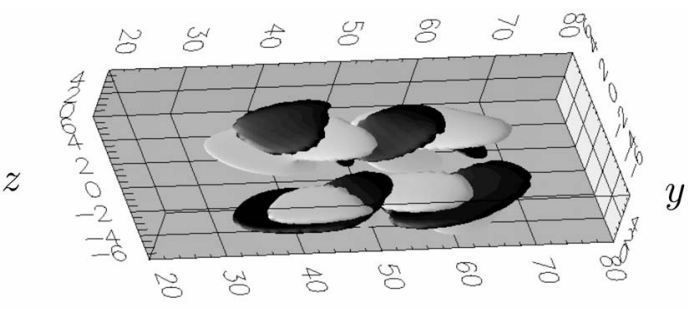

b)

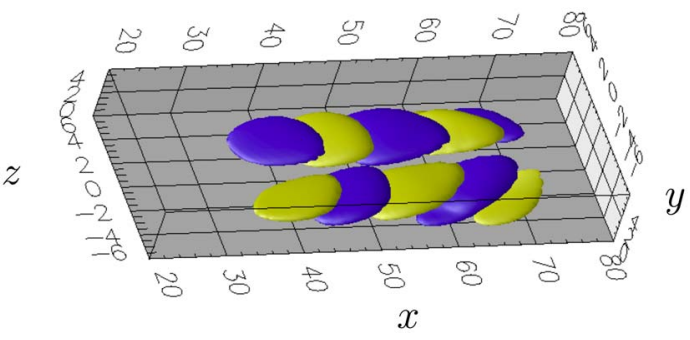

FIG. 11. (Color online) (a) Streamwise vorticity and (b) vortex tilting term for the symmetric breakdown, $\Delta \varphi=\pi$, depicted at time $t=400$. Positive and negative vorticity are represented in white and black, respectively, whereas light (gold) and dark (blue) colors are used to display surfaces of positive and negative values of the tilting. The values shown are $\omega_{x}= \pm 0.04$ and $(\partial u / \partial z)(\partial v / \partial x)-(\partial u / \partial y)(\partial w / \partial x)= \pm 0.02$. 


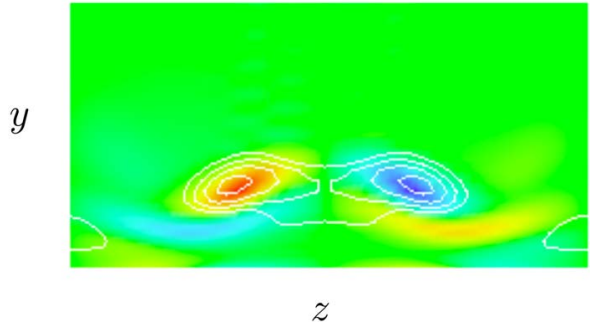

FIG. 12. (Color online) Streamwise vorticity and vortex stretching term for the symmetric breakdown, $\Delta \varphi=\pi$, depicted at time $t=450$ and $x=96$. Contour lines represent the stretching term $+\partial u / \partial x$ (contour levels 0.01), while the background color indicates the streamwise vorticity.

vorticity into vortices at the later stages of breakdown once the streak has developed significant oscillations (or splitting).

Given the resemblance with the transitional structures, one may wish to compare the vortical structures observed in the symmetric scenario with the hairpin structures observed in wall-bounded turbulence, e.g., Refs. 15, 37, and 38. The dimensions of the structures presented here can be rescaled in plus units: By averaging in space in the region of the streak interaction, a value of $\delta_{0}^{*}=14 l^{+}$is obtained; this corresponds to a streak periodicity of about $150 l^{+}$and a length of the hairpins of about $220 \mathrm{l}^{+}$, cf. Ref. 39. The structures observed in this work are very similar to those found in the studies mentioned above: Positive wall-normal velocity upstream of the spanwise vortex core, low-speed fluid between the streamwise legs of the hairpin with shear layers above them and inclination away from the wall. As in the work of Zhou et al. ${ }^{37}$ the hairpin is forming from a $\Lambda$ structure consisting of streamwise vorticity. The lift-up of the quasistreamwise vortices, formation of the shear layer, its roll-up into a spanwise vortex which bridges the two quasistreamwise legs, and the viscous vortex reconnection to form the hairpin vortex all take place in this case as well. Also the formation of the ring or $\Omega$ structure is found at later times further downstream. After this stage, the birth of a secondary hairpin is observed on the legs of a primary hairpin vortex (not shown). However, different initial conditions for the streamwise vorticity are considered here: In the present scenario, the streamwise vorticity is appearing in the form of a wave packet of positive and negative regions induced by the vortex tilting discussed above and not in a single $\Lambda$. Therefore, we notice also the appearance of the upstream $V$ structures, which, however, cannot self-sustain, and packets of hairpin structures are present already at the early stages of the breakdown. From the comparison, one can conclude that in this work, a possible mechanism for the creation of the initial condition used to study the hairpin evolution and regeneration ${ }^{37}$ is provided, which is shear instability at the streak interface. The train of hairpins observed in the experiments can be generated from the legs of a downstream structure as suggested in Ref. 38, but packets of vortices can also be induced from the beginning by the instability mechanisms responsible for the initial generation of the streamwise legs as shown here. Finally, it is important to note as in Ref. 37 that the significant role played by the mean shear suggests that the details of the hairpin regeneration process can be
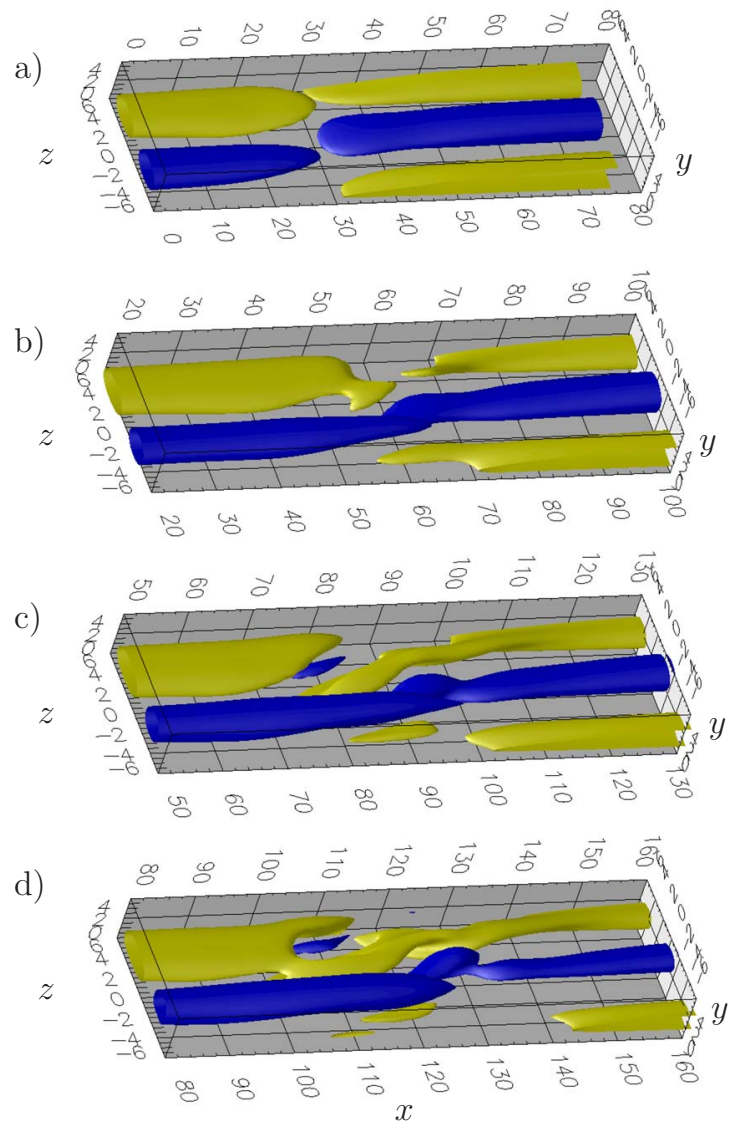

FIG. 13. (Color online) Evolution of the streaky structures during the asymmetric breakdown, $\Delta \varphi=\pi / 2$, depicted at time $t=350,400,450$, and 500 . The light (gold) color displays surfaces of positive streamwise velocity, whereas low-speed fluid is depicted in a dark (blue) color. The values shown are $u-u_{\text {blas }}= \pm 0.1$ in (a), $u-u_{\text {blas }}= \pm 0.12$ in (b) and (c), and $u-u_{\text {blas }}$ $= \pm 0.15$ in $(\mathrm{d})$.

different in laminar and turbulent boundary layers. For example, the threshold amplitude for regeneration, frequency of regeneration, and the size and shape of the resulting hairpins can be influenced by the laminar or turbulent nature of the boundary layer and parameters such as Reynolds number and streamwise pressure gradient.

\section{Asymmetric breakdown}

The breakdown process for a phase shift of $\Delta \varphi=\pi / 2$ is presented in Fig. 13 by surfaces of constant streamwise velocity perturbation. Again, high-speed fluid is indicated by a light (gold) color and low-speed fluid by a dark (blue) color. The interaction area is on the positive side of the downstream low-speed streak, i.e., on the side denoted by positive values of the spanwise coordinate $z$. In this region, where the incoming high-speed fluid is colliding with low-momentum fluid from the downstream streak, the main vorticity structures resulting from the interaction will be found. The symmetry of the breakdown for $\Delta \varphi=\pi$ (Figs. 5 and 7) is clearly broken in the present case, $\Delta \varphi=\pi / 2$. The incoming highspeed streak is not running over the low-speed region in front but is instead bending away from it moving toward the downstream region of high-speed fluid, creating what appears as a connection. Similarly, the low-speed region down- 

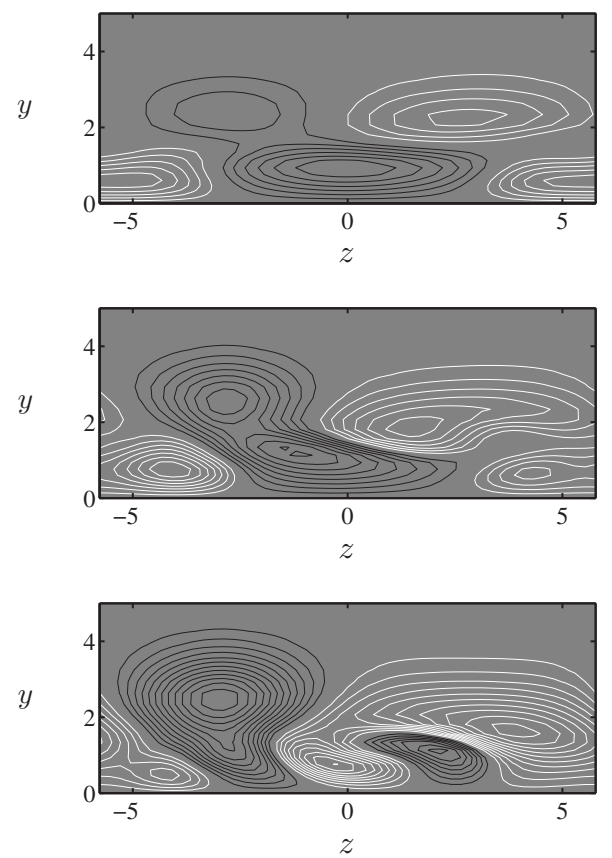

FIG. 14. The asymmetric streak interaction in the cross-stream $(y, z)$ plane. Negative (black) and positive (white) contour levels of the streamwise perturbation velocity $u-u_{\text {blas }}$ with contour spacing 0.02 are reported at time $t$ $=350,400$, and 450 and streamwise locations $x=32,60$, and 75, respectively. The initial spanwise shift between the two streaks is $\Delta \varphi=\pi / 2$.

stream is deflected toward the upstream low-speed streak. At $t=450$, the oblique regions of positive and negative velocity in the interaction area between the two streaks have started to oscillate and a patch of low-speed fluid appears within the high-speed streak connection. The length of the oscillation is about 15 and the associated structures reach a height of about 2. At later times, this oscillation grows more fierce and the amount of low-speed fluid entrained in the high-speed streak connection increases. Soon after $t=500$, this leads to turbulent breakdown. Comparing the results in Figs. 5 and 13, it can be noted that in the asymmetric case the flow structures are still relatively regular at $t=500$, indicating a slower breakdown in this case.

Cross sections, $(y, z)$ planes, of the streamwise velocity field are depicted in Fig. 14. The data are extracted at three different times and corresponding streamwise locations in the middle of the interaction area. At $t=350$, the tail of the downstream streak (close to the surface) and the head of the upstream streak (further away from the wall) are still clearly distinguishable. As the interaction region extends, the upstream high-speed fluid shifts over the downstream lowspeed fluid at $z \approx 1-2$. A surface of strong shear is thus forming: the shear is not only normal to the wall, as in the case of the symmetric breakdown, but has also a relevant component in the spanwise direction. At $t=400$, this shear surface has moved slightly toward $z=0$ and increased in strength. The high-speed fluid from the upper part of the boundary is moving downwards and reaches the wall-surface at $t \approx 450$. This leads to a splitting of the low-speed region, similar to what observed in the symmetric breakdown. However, only the lump of negative velocity on the negative- $z$ side is extending a)

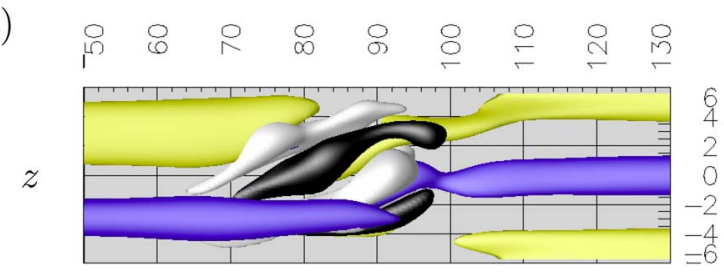

b)

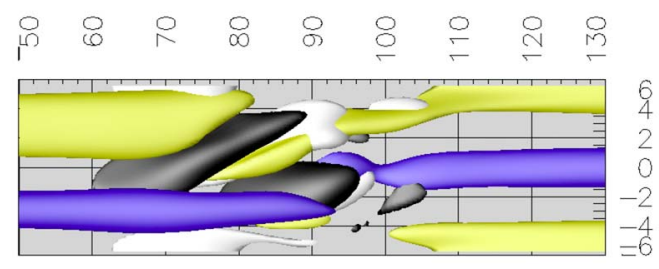

$x$

FIG. 15. (Color online) The high-frequency perturbation growing during the asymmetric streak interaction, displayed at $t=450$. In both figures, the light (gold) color displays surfaces of positive streamwise velocity $\left(u-u_{\text {blas }}\right.$ $=0.15)$, whereas low-speed fluid $\left(u-u_{\text {blas }}=-0.15\right)$ is depicted in a dark (blue) color. (a) Surface of constant wall-normal velocity perturbation $v$ $-v_{\text {blas }}= \pm 0.01$. (b) Surface of constant spanwise velocity perturbation $w$ $= \pm 0.02$, with black for negative values and white for positive values.

over the whole boundary layer; a patch of low-speed fluid remains enclosed between the wall and high-speed fluid at $z \approx 2$. On the negative side of the figures, i.e., above the center of the downstream high-speed streak, nothing relevant happens. The shear region between the tail of the downstream high-speed streak and the head of the upstream lowspeed streak is also elongating. However, this part of the interaction does not lead to breakdown.

Surfaces of constant positive and negative wall-normal and spanwise velocity perturbation, $v-v_{\text {blas }}$ and $w$, are presented in Fig. 15 together with the underlying streamwise velocity streaks. The regions of positive and negative wallnormal velocity are located on the diagonal associated with the bending of the interacting low- and high-speed streaks, with positive and negative contributions side by side on the same streak. It is clear that the most active region is on the high-speed streak connection giving longer and stronger patches of wall-normal velocity. The surfaces pertaining to the spanwise velocity perturbation are also aligned with the oscillating streaks, almost centered on them. Regions of spanwise velocity of opposite sign alternate along the underlying streak, as in the case of the sinuous streak instability, also associated with spanwise oscillations of the streak. Comparison of the relative position of the wave packets reveals the presence of a long streamwise vortex, of counterclockwise rotation, following the oscillation of the highspeed streak further downstream. The core of this vortex stretches from about $(x=65, z=-2)$ to $(x=95, z=3)$ and has a width of about 3 .

A three-dimensional view of the vortical structures characteristic of this transition scenario is reported in Fig. 16 together with the underlying streaks to indicate their relative position. The surface of negative $\lambda_{2}$ at time $t=450$ clearly shows the presence of the elongated vortex mentioned above, located on the left of the high-speed streak, positive side with 


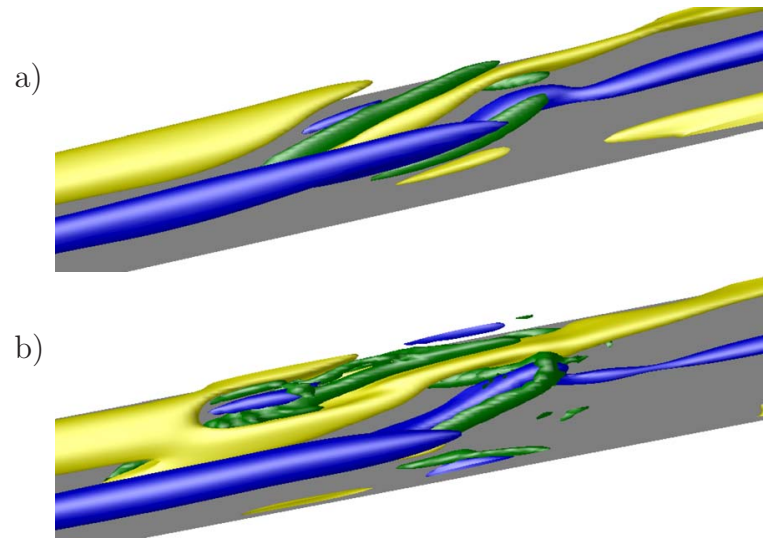

FIG. 16. (Color online) Vortical structures characteristic of the asymmetric breakdown, $\Delta \varphi=\pi / 2$, at $t=450$ and 520. In (a) dark (blue) surfaces represent low-streamwise velocity $\left(u-u_{\text {blas }}=-0.15\right)$ while light gray (gold) surfaces depict high-speed regions $\left(u-u_{\text {blas }}=0.15\right)$. Gray (green) represents negative values of $\lambda_{2}$.

reference to the increasing values of the $z$ coordinate. A second elongated structure, of opposite sign, is present on the negative side of the low-speed streaks. Both vortices are inclined upwards in the streamwise direction. A smaller structure, pointing in the negative $z$ direction, is also visible. This vortex follows the weaker oscillation of the downstream streak. Note that the pattern of bent streaks and associated vortices reported here resemble those observed in numerical studies of bypass transition ${ }^{16}$ in the case of sinuous-like breakdown. At time $t=520$, the smaller structure seems to have joined the quasi-streamwise vortex on the negative side of the low-speed streak in what appears to be a hairpin vortex, similarly to what happens to the $\Lambda$ structures in the symmetric scenario. At the same time, the long vortex following the high-speed fluid has already broken down. The low-speed fluid that is entrained in the high-speed streak leads to deformation of the $\lambda_{2}$ structures.

Analysis of the streamwise vorticity evolution reveals analogies with the case of the symmetric breakdown. A three-dimensional view of the streamwise velocity and vorticity during the interaction is displayed in Fig. 17 at times $t=350,400$, and 450 . The generation of patches of positive and negative $\omega_{x}$ following the streak oscillations can be seen. The initial production of streamwise vorticity is indeed due of vortex tilting as shown in the close-up in Fig. 18. In the plots, regions of large streamwise vorticity are reported together with those of largest tilting to show the strong spatial correlation. As in the case of $\Delta \varphi=\pi$, the tilting term $(-\partial u / \partial y)(\partial w / \partial x)$ is about one order of magnitude larger than its counterpart $(\partial u / \partial z)(\partial v / \partial x)$. As the streak oscillations grow, the term $\partial u / \partial x$ become significant and formation of streamwise vortices due to vortex stretching is found as in the symmetric scenario. A crosscut displaying the streamwise vorticity and the stretching term $\partial u / \partial x$ is reported Fig. 19 for the flow at $t=450$ and $x=93$.

Summarizing, in the case of asymmetric breakdown, the incoming streak slides along the tail of the downstream perturbation and it is deflected on the side. This leads to the formation of long quasi-streamwise vortices following the a)

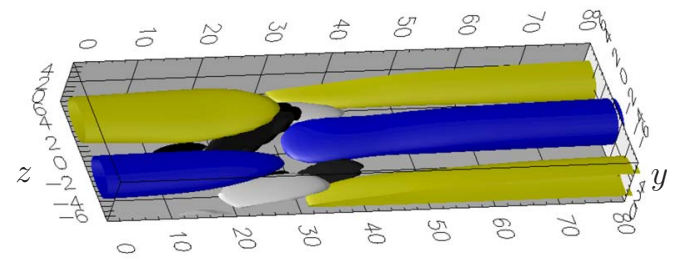

b)

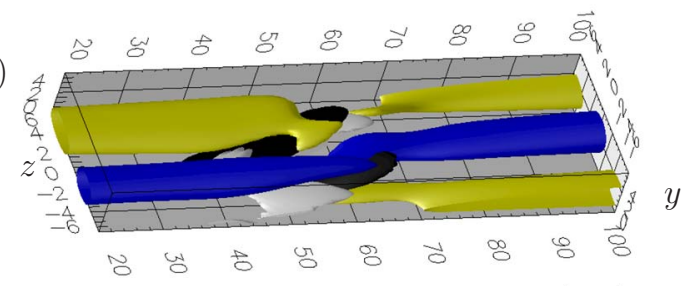

c)

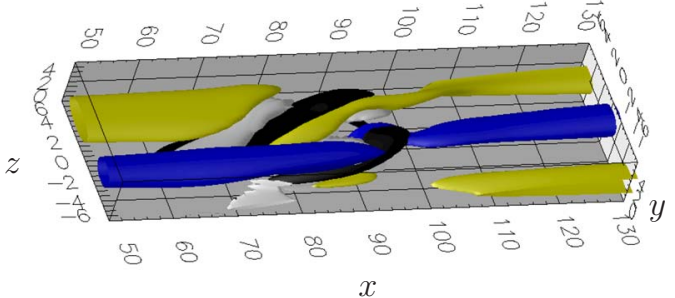

FIG. 17. (Color online) Evolution of the streamwise vorticity during the asymmetric breakdown, $\Delta \varphi=\pi / 2$, depicted at time $t=350,400$, and 450 [(a)-(c)]. Positive and negative vorticity are represented in white and black, respectively. The light (gold) color displays surfaces of positive streamwise velocity, whereas low-speed fluid is depicted in a dark (blue) color. The values shown are $\omega_{x}= \pm 0.06 ; u-u_{\text {blas }}= \pm 0.1$ in (a), $u-u_{\text {blas }}= \pm 0.12$ in (b), and $u-u_{\text {blas }}= \pm 0.15$ in (c).

streak bending. The main structure is generated by the upstream high-speed fluid. Here, the generated vorticity causes the entrainment of low-speed fluid into the high-speed region. In this region, the streak is first disrupted. Note finally that only a limited number of quasi-streamsive vortices is observed here and not a chain as in previous studies of tur-

a)

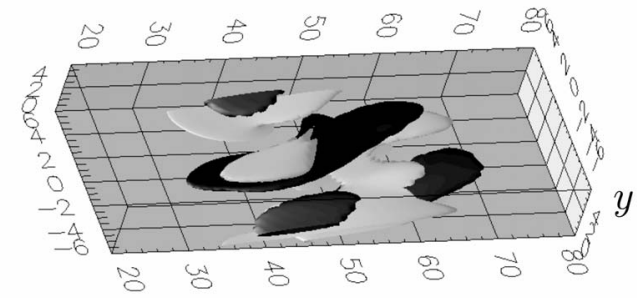

b)

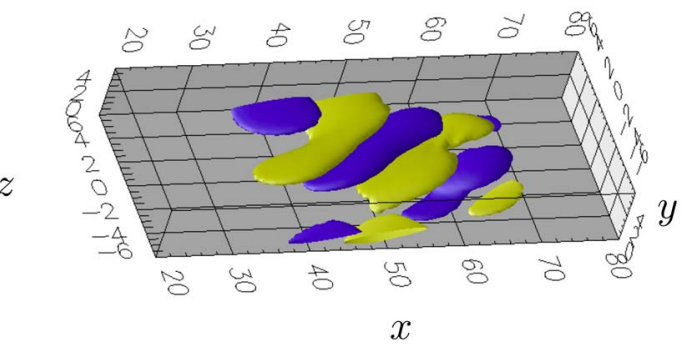

FIG. 18. (Color online) (a) Streamwise vorticity and (b) vortex tilting term for the asymmetric breakdown, $\Delta \varphi=\pi / 2$, depicted at time $t=400$. Positive and negative vorticity are represented in white and black, respectively, whereas light (gold) and dark (blue) colors are used to display surfaces of positive and negative values of the tilting. The values shown are $\omega_{x}= \pm 0.04$ and $(\partial u / \partial z)(\partial v / \partial x)-(\partial u / \partial y)(\partial w / \partial x)= \pm 0.02$. 


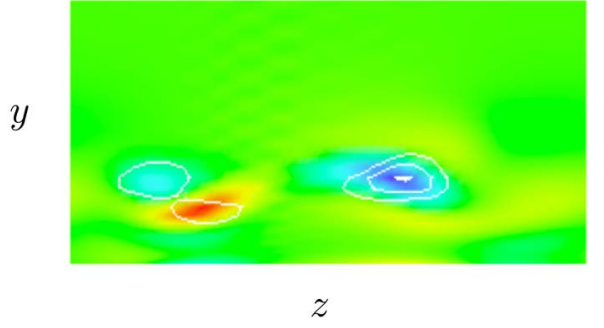

FIG. 19. (Color online) Streamwise vorticity and vortex stretching term for the asymmetric breakdown, $\Delta \varphi=\pi / 2$, depicted at time $t=450$ and $x=93$. Contour lines represent the stretching term $+\partial u / \partial x$ (contour levels 0.01 ), while the background color indicates the streamwise vorticity.

bulent flows. ${ }^{39}$ However, this is still in agreement with both the numerical simulations (Figs. 16 and 18 in Ref. 16 and Refs. 20 and 21) and experiments ${ }^{18,19}$ of bypass transition.

\section{Discussion on breakdown scenarios}

Similarities and differences between the two scenarios just presented are discussed in this section. In both cases the detrimental interaction involves the tail of a low-speed region and the head of a high-speed streak. Further, in both cases, intense localized small-scale motions are observed in the regions of strong shear created by the streak collisions. However, the spatial distribution of these surfaces of strong shear is different for the two scenarios under consideration. Surfaces of constant shear of the streamwise velocity perturbation $\mathcal{S}-\mathcal{S}_{\text {blas }}$, where $\mathcal{S}=\sqrt{(\partial u / \partial x)^{2}+(\partial u / \partial y)^{2}+(\partial u / \partial z)^{2}}$, are displayed in Figs. 20 and 21 for the symmetric and asymmetric case, respectively. In the former case, the surface of strong shear forming between the downstream low-speed streak and the upstream high-speed fluid is almost parallel to the wall, only slightly inclined upwards in the downstream direction [see Fig. 20(a)]. The main contribution to the total shear comes from the wall-normal derivative of the perturbation field. Conversely, in the case of the asymmetric breakdown, the shear surface is tilted along the streamwise axis, while still slightly pointing upwards. In this configuration, the shear has a significant component both in the wallnormal and spanwise direction [see Fig. 21(a)]. Note that the flow patterns are now viewed from the opposite side when compared to Fig. 13 and following (positive $z$ direction toward the reader) and that the shear surface leading to transition is that associated with the tail of the downstream lowspeed streak.

Before discussing the time evolution of the shear surfaces, it is useful to recall that according to classic shearlayer instability theory, production of perturbation kinetic energy is due to the work of the Reynolds stress $u u_{n}$ against the local mean shear $\partial U / \partial n$, where $n$ is the direction of the base flow velocity gradient. The instability appears as oscillations of the shear surface in the direction of the local mean velocity gradient. In the case of infinitely long streaks, the shear layer follows the cross-stream distribution of the streamwise velocity, and therefore the production zone and the associated high-frequency fluctuations appear to wrap around the boundary of the low-speed streak. ${ }^{11}$ A logical assumption
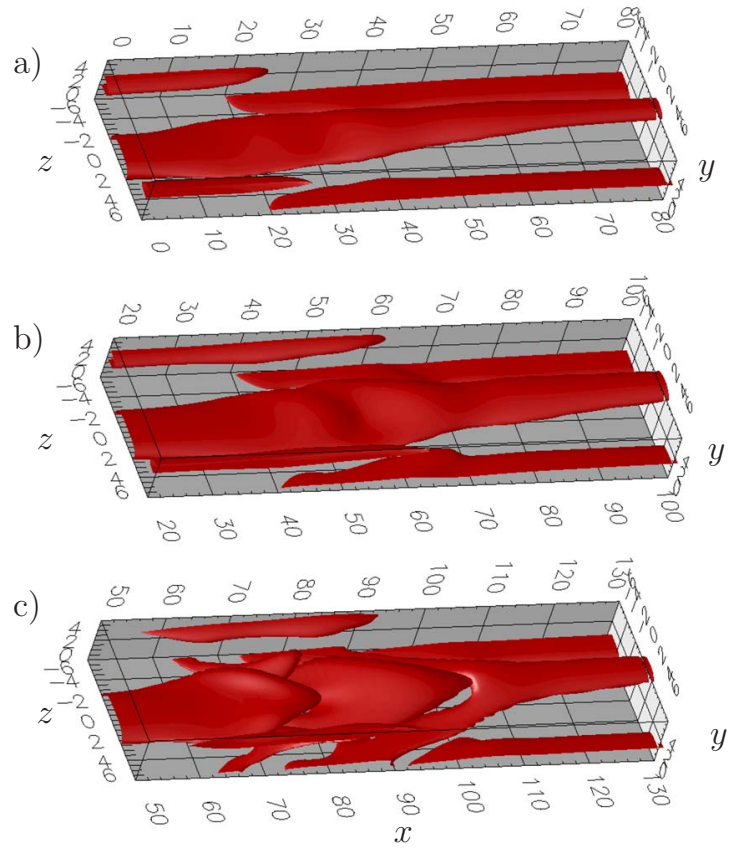

FIG. 20. (Color online) Surfaces of constant shear $\mathcal{S}-\mathcal{S}_{\text {blas, }}$ with $\mathcal{S}=\sqrt{(\partial u / \partial x)^{2}+(\partial u / \partial y)^{2}+(\partial u / \partial z)^{2}}$ at time $t=350,400$, and 450 for the symmetric breakdown, $\Delta \varphi=\pi$. The values shown are $\mathcal{S}=0.12$ in (a) and (b), whereas the surface $\mathcal{S}=0.15$ is depicted in (c).

would be to interpret the streak instability as the instability of a corrugated (in the spanwise direction) mixing layer, as done by Schoppa and Hussain. ${ }^{36}$ In the present results, the deformation of the shear surfaces at later times shows for both cases the corrugation of the surfaces in the direction
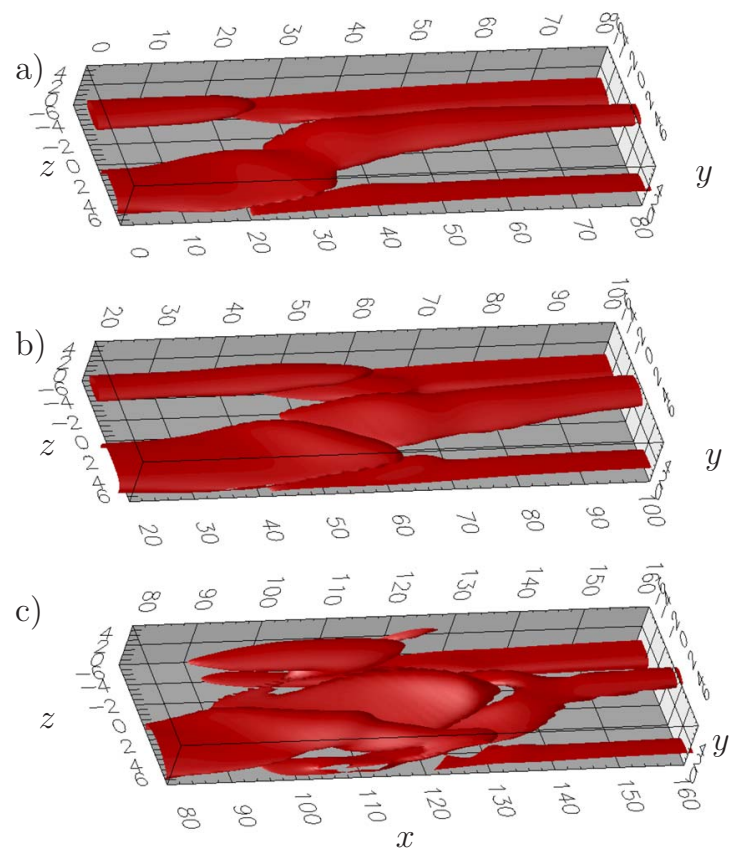

FIG. 21. (Color online) Surfaces of constant shear $\mathcal{S}-\mathcal{S}_{\text {blas }}$ at time $t=350$, 400 , and 500 for the symmetric breakdown, $\Delta \varphi=\pi / 2$. The values shown are $\mathcal{S}=0.12$ in (a) and (b), whereas the surface $\mathcal{S}=0.15$ is depicted in (c). Note that the flow pattern is now viewed from the opposite side when compared to Fig. 13 and following (positive $z$ direction toward the reader). 
normal to it [Figs. 20(b) and 21(b)]. This can be interpreted as a sign of inflectional instability of mixing-layer type (or Kelvin-Helmhotz-type) of a more complex threedimensional layer. For both cases, the shear surfaces form (at later times) isolated structures of $\Lambda$-like shape pointing downstream and tilted away from the wall [Figs. 20(c) and 21(c)]. However, in the symmetric case the structure is locally parallel to the spanwise direction, whereas in the asymmetric case essentially the same structure appears rotated in the streamwise direction, lying in the spanwise region between the oscillating high- and low-speed streak. Despite the different initial configuration, similarities are thus found between the flow structures occurring at the breakdown.

In the flow configuration under consideration, the streamwise vorticity component is initially weak and it is produced by the vortex tilting induced by the rapid streamwise variations of the cross-stream velocity components through a self-sustaining mechanism. ${ }^{35}$ These results are in agreement with previous findings in plane Couette and Poiseuille flows. Jimenez and Moin ${ }^{40}$ and Brooke and Hanratty ${ }^{41}$ found that the largest contribution to the generation of streamwise vorticity comes from the tilting term $(-\partial u / \partial y)(\partial w / \partial x)$. The latter authors computed the contribution of the term $(\partial u / \partial z)(\partial v / \partial x)$ to be less than $10 \%$ of total production. Similar results are obtained in plane Couette flow in Ref. 42. This initial growth of vorticity is associated with an increase of the streak waviness. This, in turn, induces relevant $\partial u / \partial x$ and thus stretching of the streamwise vorticity. This process leads to the quasi-streamwise vortices observed at the late stages of breakdown.

Let us finally consider the pattern of the vortical structures shown in Figs. 7 and 16. One can note that the vortices identified are elongated in the streamwise direction and follow the oscillations of the low-speed streak. All the vortices present at the breakdown stage are such that they can further sustain the streak, and the associated shear layer, through the lift-up effect. In this way, the vortices induce stronger mean shear.

From the results presented, we suggest that the streak collision scenario is associated with the formation of threedimensional shear layers due to the streak relative motion. The breakdown appears then as a mixing-layer-like instability of the surfaces of largest shear, with corrugation and disruption of the latter. The breakdown is first seen as wallnormal or spanwise oscillations of the streak, in other words as oscillation of the streak in the direction normal to the shear surfaces.

\section{E. Scenario distribution}

In the previous sections, the two different breakdown scenarios appearing when varying the relative spanwise position between straight streaks were presented. By using increments of $\pi / 12$ in the phase shift, the influence of $\Delta \varphi$ on the developing flow is examined and can be summarized as follows:

- No breakdown 0 to $5 \pi / 12$,

- Asymmetric breakdown $5 \pi / 12$ to $3 \pi / 4$,

- Symmetric breakdown $3 \pi / 4$ to $\pi$.

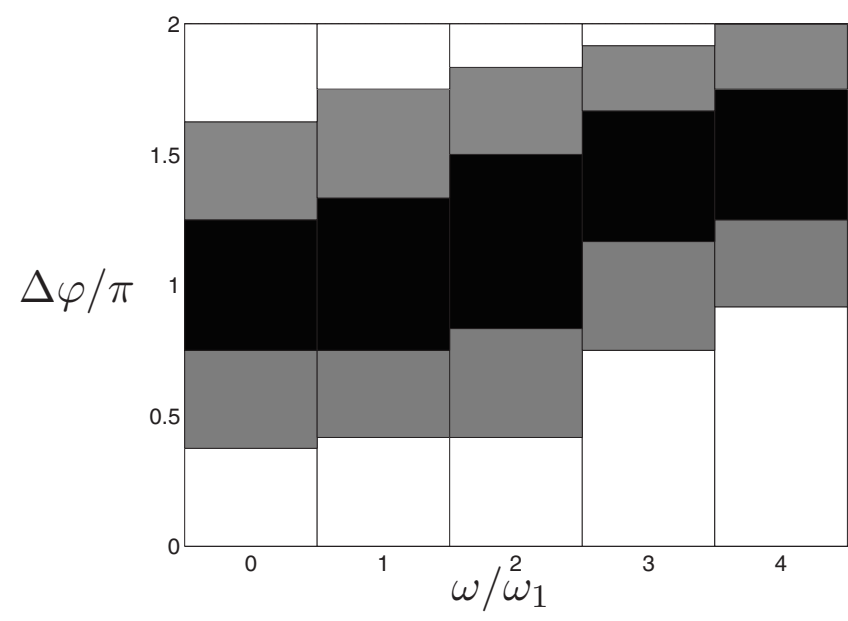

FIG. 22. Diagram of the different breakdown scenarios observed as a function of the frequency $\omega^{2}$ of the upstream streak $\left(\omega^{2}=0,1,2,3\right.$, and $\left.4 \times \omega_{1}\right)$ and of $\Delta \varphi$. White areas: No breakdown. Gray shade: Asymmetric breakdown. Black regions: Symmetric breakdown.

Two observations are in order here. First, the collision pattern is symmetric with respect to $\Delta \varphi=\pi$ for streamwisealigned streaks and therefore it is enough to consider the range $[0, \pi]$. Secondly, the breakdown is not strictly symmetric for $\Delta \varphi \neq \pi$. However, the flow structures present the same patterns as in the symmetric case, e.g., splitting of the streak and $\Lambda$ vortices, and therefore the corresponding scenario will be classified as symmetric. The results indicate that the different scenarios are associated with intervals of similar length, the asymmetric covering a range of $\Delta \varphi$ of about $2 \pi / 3$ and the symmetric of only $\pi / 2$. This would imply that the asymmetric breakdown is slightly more likely to occur if the different phase shifts are assumed to be equally probable. The type of breakdown actually observed would depend on the streak generation process and on the characteristics of the generated streaks.

\section{OBLIQUE STREAKS}

The effect of the streak spanwise motions on the interaction is now considered. At constant spanwise wavenumber, this is given by the frequency $\omega$. To examine the effect of this parameter on the collisions, simulations are carried on in which the downstream streak is still aligned with the free stream $\left(\omega^{1}=0\right)$, whereas the frequency of the upstream streak assumes the values $\omega^{2}=1,2,3$, or $4 \times \omega_{1}$, where $\omega_{1}=3.44$ $\times 10^{-3}$. The upstream inflow profile at $t=t_{\mathrm{st}}^{2}$ is set such that the maximum of the high-speed streak is at $z=0$ when the phase shift $\Delta \varphi=\pi$. It is observed that the breakdown follows the two scenarios detailed above, both a quasi-symmetric process and its asymmetric counterpart. Simulations covering the range $\Delta \varphi \in[0,2 \pi]$, with increments of $\pi / 6$ and $\pi / 12$, are thus performed to investigate whether streaks of different $\omega$ lead to a different distribution of the breakdown scenarios. The results are summarized in Fig. 22, where the different scenarios are identified by the analysis of animations of the streak collisions in a plane parallel to the wall. Symmetric breakdown is recognized when a splitting of the 
a)

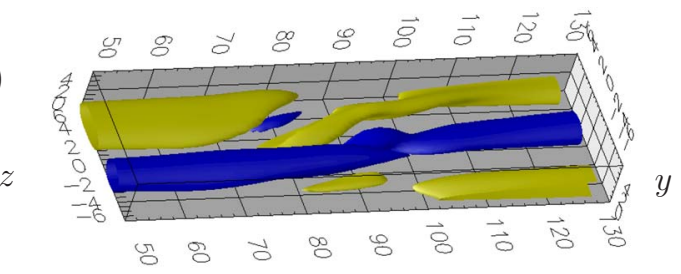

b)

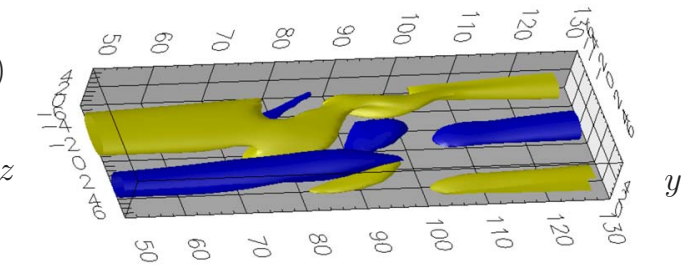

c)

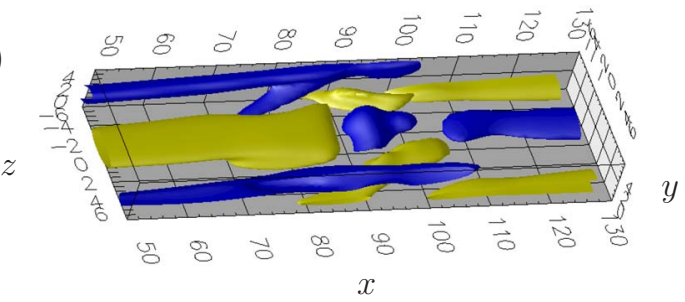

FIG. 23. (Color online) The streaky structures at breakdown for (a) $\omega=0$; (b) $\omega=2 \omega_{1}$; (c) $\omega=4 \omega_{1}$. Time $t=450$ and $\Delta \varphi=\pi / 2$. The light (gold) color displays surfaces of positive streamwise velocity, whereas low-speed fluid is depicted in a dark (blue) color. The values shown are $u-u_{\text {blas }}= \pm 0.13$ in (a) and $u-u_{\text {blas }}= \pm 0.15$ in (b) and (c).

downstream low-speed streak is present, whereas the asymmetric breakdown is characterized by spanwise streak oscillations. It can be seen in the figure that the most apparent effect of having colliding streaks at different obliqueness angles is an increase of the phase shift $\Delta \varphi$ at which the symmetric breakdown is observed. For the cases under consideration, it gradually moves from $\pi$ to $3 \pi / 2$. This can be explained by the fact that the upstream incoming streak is now moving in the spanwise direction and the collision is not happening at time $t=t_{0}$ but at later times. Therefore, in order to have a high-speed streak reaching the tail of the downstream low-speed region at the time of collision, a phase shift had to be present at the inflow. A slight increase of the range of symmetric breakdown and a small decrease of the angles leading to asymmetric breakdown can also be deduced from the figure. Simulations were also performed for interacting streaks with frequencies $\omega^{1}=-2 \omega_{1}$ and $\omega^{2}=2 \omega_{1}$, the results being almost identical to the case of $\omega^{1}=0$ and $\omega^{2}=4 \omega_{1}$.

The effect of $\omega$ on the developing breakdown structures can be illustrated by the results at $\Delta \varphi=\pi / 2$, where increasing $\omega$ leads to a slow change from asymmetric to symmetric breakdown, as seen from Fig. 22. The flow structures in terms of $u-u_{\text {blas }}$ are presented in Fig. 23 for frequencies of the second streak $\omega^{2}=0,2$, and 4 at time $t=450$. At $\omega^{2}=0$ [Fig. 23(a)], the dominant structure in the velocity distribution is that pertaining to the asymmetric breakdown discussed above. It can be seen that for higher frequencies of the upstream streak, i.e., larger spanwise velocity of the incoming streak, the head of the high-speed region has reached the tail of the downstream low-speed streak. This leads to an almost fully symmetric breakdown for $\omega=4 \omega_{1}$, as seen by the formation of a patch of low-speed fluid in combination with the upward motion on the tail of the low-speed streak in Fig. 23(c). It is also observed that as the inclination angle is increased, the effective frontal area of the secondary profile increases its spanwise extent. This induces a wider distribution of the high-speed streak of the incoming perturbation around the downstream low-speed streak and explains the observed increase in the range of phase shifts leading to the symmetric breakdown for larger $\omega$ differences.

Concluding, the results indicate that although the obliqueness of the streaky perturbations leads to a change in the shape of the interacting regions, resulting in a change of the angles of attack, the structures at the breakdown and the distribution of the different scenarios remain more or less the same.

\section{STREAK AMPLITUDE}

Finally, threshold amplitudes for the breakdown to turbulence are examined. The critical conditions identified do not include the presence of any additional noise in the flow besides the influence from the finite accuracy of the numerical computations. In a physical realization, the presence of external disturbances would most likely induce early transition and therefore the present estimate can be considered as conservative. For the results presented in this section, only steady streaks are considered, $\omega^{1}=\omega^{2}=0$, with inflow profiles yielding streak growth over a large portion of the computational domain. This would allow detrimental interactions also further downstream since it is observed that instabilities forming on streaks with decaying strength are not always leading to turbulent flow (cf. Ref. 36). The other parameters defining the streak interaction are the same as those reported in Sec. II C. It is useful to recall that critical amplitudes for the secondary instability of steady infinite streaks originating from optimally growing initial vortices such as those used here are computed in Andersson et al. ${ }^{9}$ These authors found sinuous waves to become unstable at an amplitude of $26 \%$ of the free-stream velocity, whereas varicose perturbations need streak amplitudes larger than $37 \%$. In that work, the streak amplitude is defined as one-half of the difference between the maximum and minimum of the streamwise velocity perturbation in the cross-stream plane $(y, z)$.

Results for the symmetric breakdown are presented first. In the collision scenario, with two overlapping streaks of finite length, the definition of the perturbation amplitude needs to be reconsidered. In the case of symmetric breakdown, the wall-normal maximum and minimum of the streamwise velocity perturbation in the symmetry plane are chosen to indicate the amplitude of the two colliding structures,

$$
A_{ \pm}(x)=\max _{y} / \min _{y}\left\{\left[u(x, y, 0)-u_{\text {blas }}(x, y)\right]\right\} .
$$

The spatio-temporal behavior of the amplitude of the two interacting streaks in the case of marginal conditions for the symmetric scenario is reported in Fig. 24. Marginal conditions for the formation of a region of turbulent flow are identified by varying the amplitude of the inflow perturbation by about $0.5 \%$ of the free-stream velocity. It can be seen that the interaction takes place at the tail of the downstream low- 


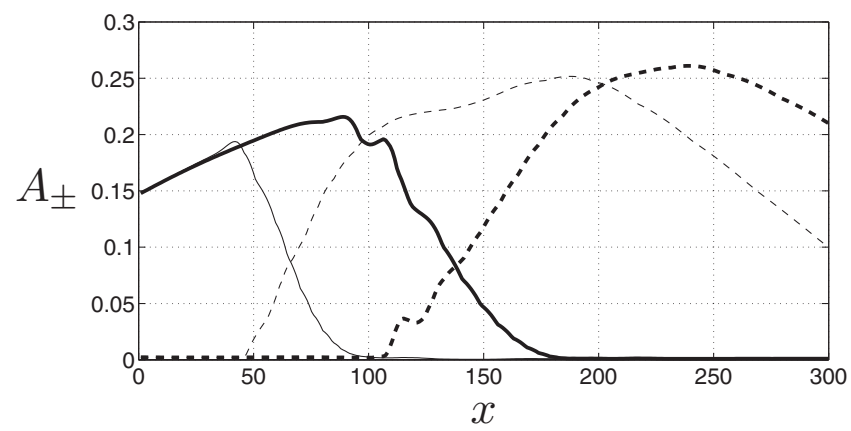

FIG. 24. Wall-normal maximum and minimum of the streamwise velocity perturbation of the colliding streaks for the symmetric breakdown in the center plane $z=0$ at $t=400$ and 500. The amplitude of the downstream lowspeed region $\left(-A_{-}\right)$is depicted by the dashed line, while the solid line is used to represent the incoming high-momentum fluid $\left(A_{+}\right)$. Thick lines indicates the perturbation at time $t=500$.

speed streak, where the incoming high-speed fluid is $0.15 U_{\infty}$ faster than the laminar boundary layer flow and the lowspeed streak in front has an amplitude of about $20 \%$ of the free-stream velocity. Oscillations of the streak amplitude, which is a sign of the developing instability, can be noticed at $t=500$. Note that in the interaction region, the maximum of the low-speed streaks is relatively close to the wall, $y$ $\approx 1$ (not shown here).

To compare the present scenario with previous studies, a local perturbation amplitude $A_{t}$ is defined as the difference between $A_{+}$and $A_{-}$. This measure indicates the total velocity difference induced by the presence of two streaks. The local critical streak amplitude for the symmetric breakdown is displayed in Fig. 25 by a solid line. The values of the velocity difference in the interaction region at the beginning of the breakdown process, $50 \lesssim x \lesssim 100$, are of about $0.18 U_{\infty}$, well below the critical threshold identified for the breakdown of a single steady streak. ${ }^{9}$ In the figure, the amplitude $A_{t}$ attained when decreasing the initial distance between the two streaks is also reported for an inflow streak amplitude below the marginal conditions for breakdown identified above $(3 \%$ lower). The gap between the two streaks is reduced by decreasing the length of the smooth tail and head of the two

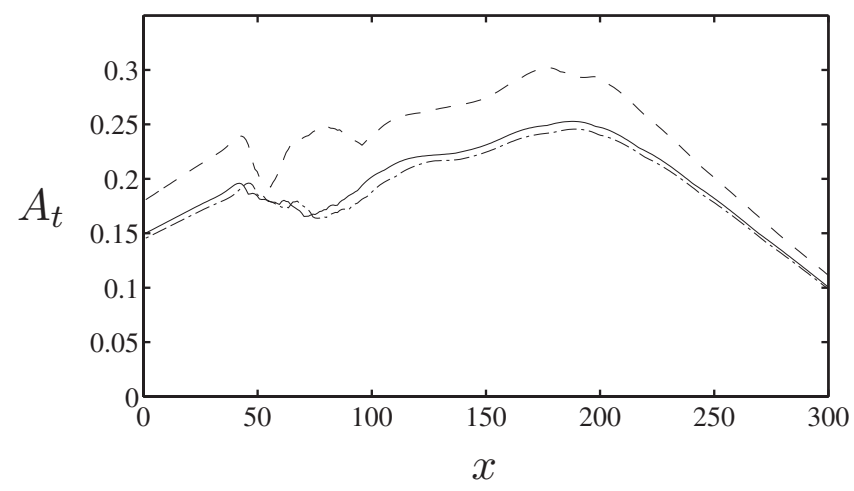

FIG. 25. Amplitude $A_{t}(x)$ of the difference between the streamwise velocity perturbation at the centers of the interacting high- and low-speed regions at marginal conditions for breakdown. Values extracted at $t=400$. (-) and $(---)$ : Symmetric scenario for different values of the initial distance between the streaks. (----): Asymmetric breakdown. colliding streaks, i.e., by reducing $t_{\text {fall }}^{1}=t_{\text {rise }}^{2}=30$ to $t_{\text {fall }}^{1}=t_{\text {rise }}^{2}$ $=15$. It can be seen that a longer overlapping region leads to local amplitudes $A_{t}$ slightly larger than the previous case, and thus to a more rapid breakdown, despite the lower amplitude of the individual streaks.

Similar analysis is repeated for the asymmetric scenario, $\Delta \varphi=\pi / 2$. In this case, the definition of a perturbation amplitude accounting for the total velocity difference induced by the two colliding streaks becomes even more difficult owing to the fully three-dimensional structure of the shear layer. Here, $A_{t}$ is still defined as the difference between the amplitude of the streamwise velocity perturbation of the upstream high-speed streak and of the downstream low-speed streak measured at their center. In the asymmetric case, the amplitude $A_{+}$is defined as the wall-normal maximum of the velocity disturbance in the plane $z=\pi /(2 \beta)$, whereas $A_{-}$still considers the low-speed region at $z=0$. Critical conditions for the asymmetric breakdown are reported in Fig. 25 for the same inflow streak profiles as in Fig. 24. The amplitude of the streamwise vortices at the inflow at which the asymmetric breakdown is first observed is about $20 \%$ larger than that necessary for a symmetric breakdown. This amounts to a velocity difference $A_{t} \approx 0.23$ at the early stages, $t=400$ and $x \in[50,100]$, of the instability. These larger initial amplitudes can be explained by the fact that in the symmetric breakdown, the velocity difference $A_{t}=A_{+}-A_{-}$is found in the $z=0$ plane, whereas in the case of the asymmetric scenario, the peak of the high-speed streak and the minimum of the low-speed streak are also misaligned in the spanwise direction and thus further apart. Therefore, by roughly estimating the shear as proportional to $A_{t} / \Delta_{s}$ with $\Delta_{s}$ the distance between the centers of the interacting streaks, it is seen that at an equal level of streak amplitude (and of $A_{t}$ ) the largest shear is forming in the case of the symmetric breakdown. The results presented here indicate the limits in the definition of the streak amplitude in the case of the proposed collision scenario. Indeed, the breakdown is related to the local shape and strength of the tail and front of the interacting structures. The necessary strength of these structures appears to be lower than for the breakdown of streaks of infinite length. This means that these streaks remain stable at their maximum amplitude and only undergo transition once they collide with another streak.

\section{CONCLUSIONS}

The present work originates from the observation that in wall-bounded shear flows with high levels of perturbations, streamwise elongated streaks are generated randomly in space and time. As the streaks grow downstream, interactions among them are likely to occur, e.g., the head of an upstream streak colliding with the tail of one downstream. Therefore, a numerical study of streak collisions is carried out, where the interacting streaks are taken to be of finite length and spanwise periodic. The results show that the collisions between two subsequent streaks can lead to breakdown without the addition of background noise. By varying the relative position of the upstream and downstream perturbation, two different scenarios are observed. In the symmet- 
ric breakdown, a frontal collision between the incoming high-speed fluid and the downstream low-speed streak is found. Conversely, in the asymmetric scenario, the upstream and downstream streaks slide along each other's side. The former scenario is characterized by the appearance at the late stages of hairpin or $\Lambda$ vortices as in the case of the varicose instability of infinite streaks, ${ }^{8}$ whereas in the asymmetric breakdown, single quasi-streamwise vortices following the bending of the streaks are identified. Symmetric and asymmetric breakdown is also observed in the numerical simulations in Refs. 20 and 21 of a boundary layer exposed to high levels of free-stream turbulence. The latter authors show the appearance of upward- and downward-moving fluid at the ends of a low-speed region leading to the formation of a hairpin structure (symmetric case) as well as the formation of one quasi-streamwise vortex in the asymmetric scenario. These data could now be interpreted in light of the proposed collision scenario.

Despite the different symmetry at the breakdown, the detrimental interaction involves for both cases the tail of a low-speed region and the head of a high-speed streak. Further, the breakdown appears in both scenarios as an instability of three-dimensional shear layers formed between the two streaks. During the breakdown, oscillations of the streaks in the direction normal to the shear surfaces are observed. In the symmetric case, wall-normal oscillations lead to splitting of the low-speed streak, whereas in the asymmetric case, the oscillations, oblique in the cross $(y, z)$ plane, induce a region of low-momentum fluid among high-speed fluid. Finally, analogies are found in the origin and evolution of the streamwise vorticity associated with the structures presented. In both scenarios, a four-step process is identified: (i) Formation of strong spanwise and wall-normal vorticity associated with the approaching streaks. (ii) Tilting of the latter in the streamwise direction by the high-frequency (fast variations in $x$ ) perturbations associated with the developing shear-layer instability. (iii) Growth of streak waviness and hence $\partial u / \partial x$. (iv) Vortex stretching by $\partial u / \partial x$. (See also Ref. 36.)

Although the present initial configuration does not contain an identifiable base flow, the similarities obtained with several studies based on the breakdown of steady streaky structures suggest that the same mechanisms are at work at the late nonlinear stages. The interaction is triggering a breakdown scenario similar to those modeled in the previous studies where, however, the streaky perturbations needed to have considerably larger amplitudes.

A parameter study of the interaction between streaks of different relative spanwise position and different obliqueness is also performed. The two different scenarios are observed in approximately equal ranges of the phase shift, where the asymmetric breakdown is slightly more likely to occur. This distribution does not change significantly for spanwise moving streaks. Threshold amplitudes for the breakdown in the absence of any additional disturbance are investigated. It is observed that the local amplitudes at the interaction location are well below the critical amplitudes found for the secondary instability of steady streaks. Interestingly, the streak amplitude at which the symmetric breakdown is first observed is about $20 \%$ lower than that necessary for an asymmetric breakdown. However, the simulations indicate that the amplitude of the streaks becomes indeed difficult to quantify in the case of collisions, since the breakdown is related to the shape of the tail and front of the interacting structures. These results imply that the two scenarios presented would be equally likely if the random distribution of relative spanwise position and amplitude were uniform.

The present work considers the formation of patches of turbulence in laminar streaky flows. However, streaks are also found to be a key element in the regeneration cycle able to sustain a turbulent flow. ${ }^{42,43}$ The collision scenario proposed here can therefore be of relevance also for turbulent wall-bounded flows, where streaky patterns are randomly induced close to the wall by the turbulence fluctuations in the outer part of the boundary layer similarly to what happens in the case of bypass transition (see the discussion in Refs. 16 and 44). Indeed, three-dimensional shear structures resembling those shown here were identified by conditional sampling from numerical simulations of turbulent channel flow in Ref. 22. Future investigations will therefore attempt to identify similar shear layers also from experimental data. The scenario proposed here can explain the birth of trains of symmetric and asymmetric hairpin vortices via the instability of high-frequency perturbations developing in the form of a wave packet, in addition to the generation from the legs of a first mature hairpin structure as suggested before. ${ }^{37}$

\section{ACKNOWLEDGMENTS}

The authors wish to thank Dr. Philipp Schlatter for many fruitful discussions and help with the flow visualizations.

${ }^{1}$ M. T. Landahl, "A note on an algebraic instability of inviscid parallel shear flows," J. Fluid Mech. 98, 243 (1980).

${ }^{2}$ P. J. Schmid and D. S. Henningson, Stability and Transition in Shear Flows (Springer, New York, 2001).

${ }^{3}$ J. D. Swearingen and R. F. Blackwelder, "The growth and breakdown of streamwise vortices in the presence of a wall," J. Fluid Mech. 182, 255 (1987).

${ }^{4}$ F. Waleffe, "Hydrodynamic stability and turbulence: Beyond transients to a self-sustaining process," Stud. Appl. Math. 95, 319 (1995).

${ }^{5}$ F. Waleffe, "On a self-sustaining process in shear flows," Phys. Fluids 9, 883 (1997)

${ }^{6}$ P. A. Elofsson, M. Kawakami, and P. H. Alfredsson, "Experiments on the stability of streamwise streaks in plane Poiseuille flow," Phys. Fluids 11, 915 (1999).

${ }^{7}$ S. C. Reddy, P. J. Schmid, J. S. Baggett, and D. S. Henningson, "On the stability of streamwise streaks and transition thresholds in plane channel flows," J. Fluid Mech. 365, 269 (1998).

${ }^{8}$ M. Asai, M. Minagawa, and M. Nishioka, "The instability and breakdown of a near-wall low-speed streak," J. Fluid Mech. 455, 289 (2002).

${ }^{9}$ P. Andersson, L. Brandt, A. Bottaro, and D. S. Henningson, "On the breakdown of boundary layers streaks," J. Fluid Mech. 428, 29 (2001).

${ }^{10}$ L. Brandt and D. S. Henningson, "Transition of streamwise streaks in zero-pressure-gradient boundary layers," J. Fluid Mech. 472, 229 (2002).

${ }^{11}$ L. Brandt, "Numerical studies of the instability and breakdown of a boundary-layer low-speed streak," Eur. J. Mech. B/Fluids 26, 64 (2007).

${ }^{12}$ W. Schoppa and F. Hussain, "Genesis and dynamics of coherent structures in near-wall turbulence, a new look," in Self-Sustaining Mechanisms of Wall Turbulence, edited by R. L. Panton (Computational Mechanics Publications, Southampton, 1997), Chap. 7, pp. 385-422.

${ }^{13}$ J. Jiménez and M. P. Simens, "Low dimensional dynamics of a turbulent wall flow," J. Fluid Mech. 435, 81 (2001).

${ }^{14}$ S. K. Robinson, "The kinematics of turbulent boundary layer structure," Technical Report TM 103859, NASA (1991).

${ }^{15}$ K. T. Christensen and R. J. Adrian, "Statistical evidence of hairpin vortex packets in wall turbulence," J. Fluid Mech. 431, 433 (2001). 
${ }^{16}$ L. Brandt, P. Schlatter, and D. S. Henningson, "Transition in boundary layers subject to free-stream turbulence," J. Fluid Mech. 517, 167 (2004).

${ }^{17}$ J. Mans, E. C. Kadijk, H. C. de Lange, and A. A. van Steenhoven, "Breakdown in boundary layer exposed to free-stream turbulence, Exp. Fluids 39, 1071 (2005).

${ }^{18}$ J. Mans, H. C. de Lange, and A. A. van Steenhoven, "Sinuous breakdown in a flat plate boundary layer exposed to free-stream turbulence," Phys. Fluids 19, 088101 (2007).

${ }^{19}$ J. Mans, "Streak development and breakdown during bypass transition," Ph.D. thesis, Technische Universiteit Eindhoven (2007).

${ }^{20}$ V. Ovchinnikov, "Numerical simulation of boundary layer transition due to external disturbances," Ph.D. thesis, University of Maryland (2006).

${ }^{21}$ V. Ovchinnikov, U. Piomelli, and M. M. Choudhari, "Numerical simulations of boundary-layer bypass transition due to high-amplitude freestream turbulence," J. Fluid Mech. (to be published).

${ }^{22}$ A. V. Johansson, P. H. Alfredsson, and J. Kim, "Evolution and dynamics of shear-layer structures in near-wall turbulence," J. Fluid Mech. 224, 579 (1991).

${ }^{23}$ M. T. Landahl, “On sublayer streaks,” J. Fluid Mech. 212, 593 (1990).

${ }^{24} \mathrm{X}$. Wu and M. Choudhari, "Linear and nonlinear instabilities of a Blasius boundary layer perturbed by streamwise vortices. Part II: Intermittent instability induced by long-wavelength Klebanoff modes," J. Fluid Mech. 483, 249 (2003)

${ }^{25}$ F. Waleffe, "Homotopy of exact coherent structures in plane shear flows," Phys. Fluids 15, 1517 (2003).

${ }^{26}$ J. Hoepffner, L. Brandt, and D. S. Henningson, "Transient growth on boundary layer streaks," J. Fluid Mech. 537, 91 (2005).

${ }^{27}$ A. Lundbladh, S. Berlin, M. Skote, C. Hildings, J. Choi, J. Kim, and D. S. Henningson, "An efficient spectral method for simulation of incompressible flow over a flat plate," Technical Report KTH/MEK/TR-99/11-SE, KTH, Department of Mechanics, Stockholm (1999).

${ }^{28}$ F. P. Bertolotti, Th. Herbert, and P. R. Spalart, "Linear and nonlinear stability of the Blasius boundary layer," J. Fluid Mech. 242, 441 (1992).

${ }^{29}$ J. Nordström, N. Nordin, and D. S. Henningson, "The fringe region technique and the Fourier method used in the direct numerical simulation of spatially evolving viscous flows," SIAM J. Sci. Comput. (USA) 20, 1365 (1999).

${ }^{30}$ M. R. Malik, T. A. Zang, and M. Y. Hussaini, "A spectral collocation method for the Navier-Stokes equations," J. Comput. Phys. 61, 64 (1985).

${ }^{31}$ O. Levin and D. S. Henningson, "Exponential vs algebraic growth and transition prediction in boundary layer flow," Flow, Turbul. Combust. 70, 183 (2003).

${ }^{32}$ M. Matsubara and P. H. Alfredsson, "Disturbance growth in boundary layers subjected to free stream turbulence," J. Fluid Mech. 430, 149 (2001).

${ }^{33}$ J. Jeong, F. Hussain, W. Schoppa, and J. Kim, "Coherent structures near the wall in a turbulent channel flow," J. Fluid Mech. 332, 185 (1997).

${ }^{34}$ V. I. Borodulin, V. R. Gaponenko, Y. S. Kachanov, D. G. W. Meyer, U. Rist, Q. X. Lian, and C. B. Lee, "Late-stage transitional boundary-layer structures: Direct numerical simulation and experiment," Theor. Comput. Fluid Dyn. 15, 317 (2002).

${ }^{35}$ G. Kawahara, J. Jiménez, M. Uhlmann, and A. Pinelli, "The instability of streaks in near-wall turbulence," Technical report, NASA-Stanford University Center for Turbulence Research, Annual Research Briefs (1998), pp. $155-170$.

${ }^{36}$ W. Schoppa and F. Hussain, "Coherent structure generation in near-wall turbulence," J. Fluid Mech. 453, 57 (2002).

${ }^{37}$ J. Zhou, R. J. Adrian, S. Balachandar, and T. M. Kendall, "Mechanisms for generating coherent packets of hairpin vortices in channel flow," J. Fluid Mech. 387, 353 (1999).

${ }^{38}$ R. J. Adrian, C. D. Meinhart, and C. D. Tomkins, "Vortex organization in the outer region of the turbulent boundary layer," J. Fluid Mech. 422, 1 (2000).

${ }^{39}$ S. K. Robinson, "Coherent motions in the turbulent boundary layer," Annu. Rev. Fluid Mech. 23, 601 (1991).

${ }^{40}$ J. Jiménez and P. Moin, "The minimal flow unit in near-wall turbulence," J. Fluid Mech. 225, 213 (1991).

${ }^{41}$ J. W. Brooke and T. J. Hanratty, "Origin of turbulence-producing eddies in a channel flow,” Phys. Fluids A 5, 1011 (1993).

${ }^{42}$ J. M. Hamilton, J. Kim, and F. Waleffe, "Regeneration mechanisms of near-wall turbulence structures," J. Fluid Mech. 287, 317 (1995).

${ }^{43}$ J. Jiménez and A. Pinelli, "The autonomous cycle of near wall turbulence," J. Fluid Mech. 389, 335 (1999).

${ }^{44}$ D. W. Wundrow and M. E. Goldstein, "Effect on a laminar boundary layer of small-amplitude streamwise vorticity in the upstream flow," J. Fluid Mech. 426, 229 (2001). 